Research Article

\title{
Uniformly Convergent Numerical Scheme for Singularly Perturbed Parabolic PDEs with Shift Parameters
}

\author{
Mesfin Mekuria Woldaregay $\mathbb{D i D}^{1}$ and Gemechis File Duressa $\left.{ }^{2}\right)^{2}$ \\ ${ }^{1}$ Department of Applied Mathematics, Adama Science and Technology University, Adama, Ethiopia \\ ${ }^{2}$ Department of Mathematics, Jimma University, Jimma, Ethiopia \\ Correspondence should be addressed to Mesfin Mekuria Woldaregay; msfnmkr02@gmail.com
}

Received 23 January 2021; Revised 7 April 2021; Accepted 12 November 2021; Published 30 November 2021

Academic Editor: Jean Jacques Loiseau

Copyright ( 2021 Mesfin Mekuria Woldaregay and Gemechis File Duressa. This is an open access article distributed under the Creative Commons Attribution License, which permits unrestricted use, distribution, and reproduction in any medium, provided the original work is properly cited.

\begin{abstract}
In this article, singularly perturbed parabolic differential difference equations are considered. The solution of the equations exhibits a boundary layer on the right side of the spatial domain. The terms containing the advance and delay parameters are approximated using Taylor series approximation. The resulting singularly perturbed parabolic PDEs are solved using the Crank-Nicolson method in the temporal discretization and nonstandard finite difference method in the spatial discretization. The existence of a unique discrete solution is guaranteed using the discrete maximum principle. The uniform stability of the scheme is investigated using solution bound. The uniform convergence of the scheme is discussed and proved. The scheme converges uniformly with the order of convergence $O\left(N^{-1}+(\Delta t)^{2}\right)$, where $N$ is number of subintervals in spatial discretization and $\Delta t$ is mesh length in temporal discretization. Two test numerical examples are considered to validate the theoretical findings of the scheme.
\end{abstract}

\section{Introduction}

Differential equations play a prominent role in many disciplines for modelling real-world problems in which the evolution of the system depends on the present state of the system. Problems that depend on the past and current evolution of the system are modelled by the delay deferential equation [1].

Singularly perturbed differential equations (SPDEs) are differential equations in which their highest order derivative term is multiplied by a small perturbation parameter. Such types of differential equations model various processes in applied mathematics, for instance, in fluid mechanics, fluid dynamics, elasticity, quantum mechanics, chemical-reactor theory, reaction-diffusion processes, and other domains of fluid motion [2, 3]. Singularly perturbed differential difference equations model physical problems for which the evaluation does not only depend on the present state of the system but also on the past history. In general, when the perturbation parameter tends to zero, the smoothness of the solution of the singularly perturbed differential difference equations deteriorates and it forms a boundary layer [4]. Such types of singularly perturbed differential difference equations have an application in the modelling of neuronal variability $[5,6]$.

Musila and Lansky [7] give a generalization for the model in $[5,6]$ in the form of singularly perturbed parabolic differential difference equations (SPPDDEs):

$$
\begin{aligned}
u_{t}(x, t)= & \frac{\sigma^{2}}{2} u_{x x}(x, t)+\left(\mu_{D}-\frac{x}{\tau}\right) u_{x}(x, t)+\lambda_{s} u\left(x+a_{s}, t\right) \\
& +\omega_{s} u\left(x+i_{s}, t\right)-\left(\lambda_{s}+\omega_{s}\right) u(x, t),
\end{aligned}
$$

for studying time evolution of the trajectories of the membrane potential, where the first derivative term denotes the exponential decay between two consecutive jumps caused by the input processes. The membrane potential 
decays exponentially to the resting level with a membrane time constant $\tau$. $\mu_{D}$ and $\sigma$ are diffusion moments of the Wiener process characterizing the influence of dendritic synapses on the cell excitability. The excitatory input contributes to the membrane potential by an amplitude $a_{s}$ with intensity $\lambda_{s}$, and similarly, the inhibitory input contributes by an amplitude $i_{s}$ with intensity $\omega_{s}$.

The mathematical model in (1) is in the form of SPPDDEs. There are no known analytical methods of solution for physiologically acceptable parameters [7]. So, to simulate the solution of this model, one has to develop suitable numerical methods. Numerical methods developed for regular problems become inapplicable for solving SPPDDEs when the perturbation parameter tends to zero [8]. Due to this, there is an interest in the development of numerical methods that converges uniformly (or converges independent of the perturbation parameter).

In recent years, scholars have devoted for the numerical treatment of SPPDDEs. In a series of papers [8-11], Bansal and Sharma developed different numerical schemes for solving SPPDDEs with large shift arguments. Kumar and Kadalbajoo [12] applied the implicit Euler method in temporal discretization and B-spline collocation method on the Shishkin mesh in the spatial discretization, and they obtain a first-order uniformly convergent result. Ramesh and Kadalbajoo [4] used the implicit Euler method in temporal discretization and upwind and midpoint upwind FDM on Shishkin mesh for the spatial discretization. They obtain almost first-order uniformly convergent numerical result. Rao and Chakravarthy [13] applied the implicit Euler method in temporal discretization and exponentially fitted operator FDM in the spatial discretization. They obtain a first-order uniformly convergent result. Woldaregay and Duressa [14-16] developed numerical schemes using fitted operator methods on uniform mesh. They obtained first-order uniformly convergent result. Kumar et al. [17] developed stable FDM using the implicit Euler method for temporal discretization and a class of nonstandard FDM using infinite serious approximation for the derivative terms in spatial discretization, and they apply Richardson extrapolation technique to extend the rate of convergence of the scheme to order two. Shivhare et al. [18] developed a numerical scheme using the implicit Euler method in temporal discretization and quadratic B-spline collocation method for the spatial discretization on the exponentially graded mesh. Daba and Duressa [19] used the implicit Euler method in temporal discretization and extended cubic B-spline collocation method for the spatial discretization on the Shishkin mesh.

In this article, we developed numerical scheme using the Crank-Nicolson method in the temporal discretization and nonstandard FDM in the spatial discretization. We used Rothe's procedure for discretizing the problem (i.e., first we discretize the temporal direction and followed by spatial discretization). The proposed scheme satisfies the discrete maximum principle and uniform stability estimate. The developed scheme treats the considered problem accurately with parameter uniform convergence.
1.1. Organization of the Paper. The rest of the article is organized as follows. In Section 2, the statement of the problem is given. In Section 3, the asymptotic approximation and its properties are discussed. In Section 4, the proposed numerical scheme and its uniform convergence analysis are given. In Section 5, numerical examples, results, and discussions are given. Finally, in Section 6, the conclusion of the article is included.

1.2. Notation. In this article, $N$ and $M$ are denoted for the number of mesh points in spatial and temporal discretization, respectively. The symbol $C$ (in some cases indexed) denotes a positive constant independent of the perturbation parameter $\varepsilon$ and $N$. The norm $\|$.$\| denotes the supremum (or$ maximum) norm.

\section{Statement of the Problem: Aim of the Research}

On the domain $D=\Omega \times \Lambda=(0,1) \times(0, T]$, for some fixed number $T>0$, with the boundary $\partial D=\bar{D} \backslash D$, a singularly perturbed parabolic differential difference equation is given by

$$
\left(\frac{\partial}{\partial t}+L_{\varepsilon, \delta, \eta}\right) u(x, t)=f(x, t), \quad(x, t) \in D
$$

where

$$
\begin{aligned}
L_{\varepsilon, \delta, \eta} u(x, t)= & -\varepsilon^{2} u_{x x}(x, t)+a(x) u_{x}(x, t) \\
& +\alpha(x) u(x-\delta, t)+\beta(x) u(x, t) \\
& +\omega(x) u(x+\eta, t),
\end{aligned}
$$

$\varepsilon, 0<\varepsilon \ll 1$ is a singular perturbation parameter and $\delta$ and $\eta$ are small delay and advance parameters satisfying $\delta, \eta=o(\varepsilon)$, with the initial and interval conditions

$$
\begin{aligned}
& u(x, 0)=u_{0}(x), \quad x \in D_{b}=\{(x, 0): x \in \bar{\Omega}\}, \\
& u(x, t)=\phi(x, t), \quad(x, t) \in D_{l}=\{(x, t):(x, t) \in[-\delta, 0] \times \Lambda\}, \\
& u(x, t)=\psi(x, t), \quad(x, t) \in D_{r}=\{(x, t):(x, t) \in[1,1+\eta] \times \Lambda\} .
\end{aligned}
$$

The problem in (2) and (4) is the simplified form of the model in (1) by including the source function $f$ with specified initial and interval conditions. From the convection diffusion fluid flow point of view, the terms in (2) and (4) can be classified as $-\varepsilon^{2}\left(\partial^{2} / \partial x^{2}\right) u(x, t)$ is called the diffusion term; $(\partial / \partial t+a(x) \partial / \partial x) u(x, t)$ is called the convection term and the nonderivative terms referred to as the reaction terms.

For the existence of a unique solution, the functions $a, \alpha$, $\beta, \omega, f, u_{0}, \phi$, and $\psi$ are assumed to be sufficiently smooth, bounded, and independent of $\varepsilon$. The coefficient functions $\alpha, \beta$, and $\omega$ are assumed to satisfy

$$
\alpha(x)+\beta(x)+\omega(x) \geq \zeta>0, \quad x \in \bar{\Omega},
$$

for some constant $\zeta$. For small values of $\varepsilon$, the solution of (2) and (4) exhibits left or right boundary layer depending on 
the sign of the coefficient function of the convection term $a(x)$. If $a(x)<0$, a regular boundary layer occurs in the neighbourhood of $D_{l}$, and for the case $a(x)>0$, the layer occurs in the neighbourhood of $D_{r}$ [20].

The main contribution of this article is, to develop uniformly convergent numerical scheme using nonstandard FDM for solving the problem in (2) and (4). The developed method is applicable on the uniform mesh.

\section{Properties of the Analytical Solution}

In this section, we discuss behaviours of the analytical solution of the problem in (2) and (4). When the shift parameters $\delta$ and $\eta$ are smaller than $\varepsilon$, using Taylor series approximation for the terms with shifts is valid [21]. So, we approximate $u(x-\delta, t)$ and $u(x+\eta, t)$ as

$$
\begin{aligned}
& u(x-\delta, t) \approx u(x, t)-\delta u_{x}(x, t)+\left(\frac{\delta^{2}}{2}\right) u_{x x}(x, t)+O\left(\delta^{3}\right), \\
& u(x+\eta, t) \approx u(x, t)+\eta u_{x}(x, t)+\left(\frac{\eta^{2}}{2}\right) u_{x x}(x, t)+O\left(\eta^{3}\right) .
\end{aligned}
$$

Using the approximations in (6) into (2), we obtain

$$
\left(\frac{\partial}{\partial t}+L_{c_{\varepsilon}}\right) u(x, t)=f(x, t), \quad(x, t) \in D
$$

with initial and boundary conditions

$$
\begin{aligned}
& u(x, 0)=u_{0}(x), \quad x \in D_{b}=\{(x, 0): x \in \bar{\Omega}\}, \\
& u(0, t)=\phi(0, t), \quad(x, t) \in D_{l}=\{(x, t): x=0, t \in \Lambda\}, \\
& u(1, t)=\psi(1, t),(x, t) \in D_{r}=\{(x, t): x=1, t \in \Lambda\},
\end{aligned}
$$

where $\quad L_{c_{\varepsilon}} u(x, t)=-c_{\varepsilon}(x) u_{x x}(x, t)+p(x) u_{x}(x, t)+q(x) u$ $(x, t), c_{\varepsilon}(x)=\varepsilon^{2}-\alpha(x)\left(\delta^{2} / 2\right)-\omega(x)\left(\eta^{2} / 2\right), p(x)=a(x)-$ $\delta \alpha(x)+\eta \omega(x)$ and $q(x)=\alpha(x)+\beta(x)+\omega(x)$.

For small values of the shift parameters $\delta, \eta,(2)$ and (5) and (7) and (8) are asymptotically equivalent, since the difference between the two equations is $O\left(\delta^{3}, \eta^{3}\right)$. We assumed that $0<c_{\varepsilon}(x) \leq \varepsilon^{2}-\delta^{2}(\alpha / 2)-\eta^{2}(\omega / 2)=c_{\varepsilon}$, where $\alpha$ and $\beta$ are the lower bounds for $\alpha(x)$ and $\omega(x)$, respectively. Furthermore, we assumed $p(x) \geq p^{*}>0$, which implies the occurrence of the boundary layer in the neighbourhood of $D_{r}$. The other case $p(x) \leq p^{*}<0$ implies the occurrence of the boundary layer in the neighbourhood of $D_{l}$ and can be treated similarly. The layer is maintained for sufficiently small shift parameters $\delta, \eta$.

Lemma 1. To avoid the presence of a singularity in the numerical solution, it is required that the given data satisfy the compatibility conditions $u_{0}(0)=\phi(0,0), u_{0}(1)=\psi(1,0)$, and

$$
\begin{aligned}
& \phi_{t}(0,0)-c_{\varepsilon}\left(u_{0}\right)_{x x}(0)+p(0)\left(u_{0}\right)_{x}(0)+q(0) u_{0}(0)=f(0,0), \\
& \psi_{t}(1,0)-c_{\varepsilon}\left(u_{0}\right)_{x x}(1)+p(1)\left(u_{0}\right)_{x}(1)+q(1) u_{0}(1)=f(1,0),
\end{aligned}
$$

so that the data match at the two corners $(0,0)$ and $(1,0)$. Let the functions $p(x), q(x)$, and $f(x, t)$ be continuous on $D$, then (7) and (8) have a unique solution $u \in C^{2}(D)$. In particular, when the compatibility conditions are not satisfied, a unique classical solution still exist but may not be differentiable on all of $\partial D$ [22].

In this article, we considered the case, when boundary layer occurs in the neighbourhood of $D_{r}$. Using the compatibility conditions, we deduce that there exists a constant $C$ independent of $c_{\varepsilon}$ such that for all $(x, t) \in \bar{D}$.

$$
\begin{aligned}
|u(x, t)-u(x, 0)| & =\left|u(x, t)-u_{0}(x)\right| \leq C t, \\
|u(x, t)-u(0, t)| & =|u(x, t)-\phi(0, t)| \leq C(1-x) .
\end{aligned}
$$

For details, interested readers can refer [22].

Remark 1. Note that there does not exist a constant $C$ independent of $c_{\varepsilon}$ such that $|u(x, t)-\psi(1, t)| \leq C x$, since the layer occurs in the neighbourhood of $D_{r}$.

The problem obtained by setting $c_{\varepsilon}=0$ in (7) and (8) is called reduced problem and given as

$$
u_{t}^{0}(x, t)+p(x) u_{x}^{0}(x, t)+q(x) u^{0}(x, t)=f(x, t), \quad(x, t) \in D,
$$

with the initial conditions

$$
u^{0}(x, 0)=u_{0}(x), \quad x \in \bar{\Omega}, u^{0}(0, t)=\phi(0, t), t \in \bar{\Lambda} .
$$

It is first-order hyperbolic PDEs with initial data given along sides $t=0$ and $x=0$ of the domain $\bar{D}$. For small values of $c_{\varepsilon}$, the solution $u(x, t)$ of the problem in (7) and (8) is very close to the solution $u^{0}(x, t)$ of (11) and (12).

Lemma 2. The maximum principle. Let $z$ be any sufficiently smooth function defined on $D$, which satisfies $z(x, t) \geq 0,(x, t) \in \partial D$. Then, $\quad\left(\partial / \partial t+L_{c_{\varepsilon}}\right) z(x, t)>0$, $(x, t) \in D$ implies that $z(x, t) \geq 0, \forall(x, t) \in \bar{D}$.

Proof. Let $\left(x^{*}, t^{*}\right)$ be such that $z\left(x^{*}, t^{*}\right)=\min _{(x, t) \in \bar{D}} z(x, t)$ and suppose that $z\left(x^{*}, t^{*}\right)<0$. It is clear that $\left(x^{*}, t^{*}\right) \notin \partial D$ implying that $\left(x^{*}, t^{*}\right) \in D$. Since $z\left(x^{*}, t^{*}\right)=\min _{(x, t) \in \bar{D}} z$ $(x, t)$ from the theory of extrema of a function in calculus implies, $z_{x}\left(x^{*}, t^{*}\right)=0, z_{t}\left(x^{*}, t^{*}\right)=0$ and $z_{x x}\left(x^{*}, t^{*}\right) \geq 0$ giving that $\left(\partial / \partial t+L_{c}\right) z\left(x^{*}, t^{*}\right)<0$, which is contradiction to the assumption. Therefore, $\left((\partial / \partial t)+L_{c_{\varepsilon}}\right) z\left(x^{*}, t^{*}\right)>0$, $(x, t) \in D$. Hence, we obtain $z(x, t) \geq 0, \forall(x, t) \in \bar{D}$.

Lemma 3. Uniform stability estimate. Let $u(x, t)$ be the solution of the problem in (7) and (8). Then, it satisfies the following bound:

$$
|u(x, t)| \leq \zeta^{-1}\|f\|+\|u\|_{\partial D},
$$

where $\|u\|_{\partial D}=\max \left\{\left|u_{0}(x)\right|,|\phi(0, t)|,|\psi(1, t)|\right\}$.

Proof. The proof is by the construction of barrier functions $\vartheta^{ \pm}(x, t)$ as $\vartheta^{ \pm}(x, t)=\zeta^{-1}\|f\|+\|u\|_{\partial D} \pm u(x, t)$, and applying the maximum principle, we obtain the required bound. For 
the detail of the proof, the interested reader can refer [16].
Lemma 4. Let $u(x, t)$ be solution of (7) and (8). Then, the derivatives of the solutions with respect to $x$ and $t$ satisfy following the bound:

$$
\begin{aligned}
& \left|\frac{\partial^{k}}{\partial x^{k}} u(x, t)\right| \leq C\left(1+c_{\varepsilon}^{-k} \exp \left(\frac{-p^{*}(1-x)}{c_{\varepsilon}}\right)\right), \quad(x, t) \in \bar{D}, 0 \leq k \leq 4, \\
& \left|\frac{\partial^{l}}{\partial t^{l}} u(x, t)\right| \leq C,(x, t) \in \bar{D}, \quad 0 \leq l \leq 2,
\end{aligned}
$$

where $p^{*}$ is lower bound of $p(x)$.

Proof. Refer in [23].

\section{Formulation of the Numerical Scheme}

4.1. Temporal Discretization. We discretize the temporal domain $[0, T]$ into $M$ subintervals using the grid points $t_{0}=0, t_{j}=j \Delta t, j=0,1,2, \ldots, M$, where $\Delta t=T / M$. Let $U_{j+1}(x)$ is denoted for the approximation of $u\left(x, t_{j+1}\right)$ at the $(j+1)$ th time level discretization. The continuous problem in (7) and (8) semidiscretized using the Crank-Nicolson method in the temporal direction resulting to a system of boundary value problems

$$
L_{c_{\varepsilon}}^{\Delta t} U_{j+1}(x)=g_{j+1}(x), \quad x \in \bar{\Omega}, j=1,2, \ldots, M-1,
$$

with the following discretized boundary conditions:

$$
U_{j+1}(0)=\phi\left(0, t_{j+1}\right), U_{j+1}(1)=\psi\left(1, t_{j+1}\right),
$$

where, $\quad L_{c_{\varepsilon}}^{\Delta t} U_{j+1}(x)=-\left(c_{\varepsilon} / 2\right)\left(U_{j+1}(x)\right)_{x x}+p(x) / 2\left(U_{j+1}\right.$ $(x))_{x}+d(x) U_{j+1}(x)$ and $g_{j+1}(x)=c_{\varepsilon} / 2\left(U_{j}(x)\right)_{x x}-p(x) / 2$ $\left(U_{j}(x)\right)_{x}-r(x) U_{j}(x)+1 / 2\left[f\left(x, t_{j+1}\right)+f\left(x, t_{j}\right)\right]$, for $d(x)=1 / \Delta t+q(x) / 2>0$ and $r(x)=q(x) / 2-1 / \Delta t$.

Lemma 5. Semidiscrete maximum principle. Let $z_{j+\underline{1}}$ be a sufficiently smooth function on the domain $\bar{\Omega}$. If $z_{j+1}(0) \geq 0, z_{j+1}(1) \geq 0$, and $L_{x, c_{\varepsilon}}^{\Delta t} z_{j+1}(x) \geq 0, x \in \Omega$, then, $z_{j+1}(x) \geq 0, \forall x \in \bar{\Omega}$.

Proof. Let $x^{*} \in \bar{\Omega}$ be such that $z_{j+1}\left(x^{*}\right)=\min _{x \in \bar{\Omega}} z_{j+1}(x)$ and suppose that $z\left(x^{*}\right)<0$. From the assumption, it is clear that $x^{*} \notin\{0,1\}$, which implies that $x^{*} \in(0,1)$. Now, using the differential operator at the point $x^{*}$, we have

$$
\begin{aligned}
L_{x, c_{\varepsilon}}^{\Delta t} z_{j+1}\left(x^{*}\right)= & -\frac{c_{\varepsilon}}{2}\left(z_{j+1}\left(x^{*}\right)\right)_{x x}+\frac{p\left(x^{*}\right)}{2}\left(z_{j+1}\left(x^{*}\right)\right)_{x} \\
& +d\left(x^{*}\right) z_{j+1}\left(x^{*}\right) .
\end{aligned}
$$

Applying the property in calculus for minimum points, we obtain

$$
\begin{gathered}
\left(z_{j+1}\left(x^{*}\right)\right)_{x}=0, \\
\left(z_{j+1}\left(x^{*}\right)\right)_{x x} \geq 0 .
\end{gathered}
$$

Using the estimates in (18) into (17), we obtain $L_{x, \mathcal{C}_{\varepsilon}}^{\Delta t} z_{j+1}\left(x^{*}\right)<0$, which is contradiction to the assumption $L_{x, c_{\varepsilon}}^{\Delta t} z_{j+1}\left(x^{*}\right) \geq 0, x \in \Omega$. Therefore, we conclude that $z_{j+1}(x) \geq 0, \forall x \in \bar{\Omega}$.

Next, let us analyse the bound of the error in temporal semidiscretization. Let us denote $u\left(x, t_{j+1}\right)$ and $U_{j+1}(x)$ be the exact and approximate solution of the problem in (7) and (8) using the above discretization. Let us denote the local error for each time step by $e_{j+1}:=u\left(x, t_{j+1}\right)-U_{j+1}(x)$.

Lemma 6. Local error estimate. Suppose that $\left|\left(\partial^{l} / \partial t^{l}\right) u(x, t)\right| \leq C, 0 \leq l \leq 2$. The local error estimate in the temporal discretization is given by

$$
\left\|e_{j+1}\right\| \leq C_{1}(\Delta t)^{3} \text {. }
$$

Proof. Using Taylor's series expansion for $u\left(x, t_{j+1}\right)$ and $u\left(x, t_{j}\right)$ centered at $t_{j+(1 / 2)}$, we obtain

$$
\begin{aligned}
u\left(x, t_{j+1}\right) & =u\left(x, t_{j+(1 / 2)}\right)+\frac{\Delta t}{2} u_{t}\left(x, t_{j+(1 / 2)}\right)+\frac{(\Delta t)^{2}}{8} u_{t t}\left(x, t_{j+(1 / 2)}\right)+O\left(\Delta t^{3}\right), \\
u\left(x, t_{j}\right) & =u\left(x, t_{j+(1 / 2)}\right)-\frac{\Delta t}{2} u_{t}\left(x, t_{j+(1 / 2)}\right)+\frac{(\Delta t)^{2}}{8} u_{t t}\left(x, t_{j+(1 / 2)}\right)+O\left(\Delta t^{3}\right) .
\end{aligned}
$$


Substituting (20) into (7), we obtain

$$
\begin{aligned}
\frac{u\left(x, t_{j+1}\right)-u\left(x, t_{j}\right)}{\Delta t}= & u_{t}\left(x, t_{j+(1 / 2)}\right)+O\left((\Delta t)^{2}\right) \\
= & c_{\varepsilon} u_{x x}\left(x, t_{j+(1 / 2)}\right)-p(x) u_{x}\left(x, t_{j+(1 / 2)}\right)-q(x) u\left(x, t_{j+(1 / 2)}\right)+f\left(x, t_{j+(1 / 2)}\right) \\
& +O\left((\Delta t)^{2}\right),
\end{aligned}
$$

where, $u\left(x, t_{j+(1 / 2)}\right)=1 / 2\left[u\left(x, t_{j+1}\right)+u\left(x, t_{j}\right)\right]+O\left(\Delta t^{2}\right)$, $f\left(x, t_{j+1 / 2}\right)=1 / 2\left[f\left(x, t_{j+1}\right)+f\left(x, t_{j}\right)\right]+O\left(\Delta t^{2}\right)$.

Now, it can be seen that the local error is the solution of

$$
\begin{aligned}
L_{x, c_{\varepsilon}}^{\Delta t} e_{j+1} & =O\left(\Delta t^{3}\right), \\
e_{j+1}(0) & =0=e_{j+1}(1) .
\end{aligned}
$$

Hence, applying the maximum principle on the operator gives

$$
\left\|e_{j+1}\right\| \leq C_{1}(\Delta t)^{3} \text {. }
$$

Next, we need to show the bound for the global error of the temporal semidiscretization. Let us denote $E_{j+1}$ for the global error estimate up to the $(j+1)$ th time step.

Lemma 7. Global error estimate. The global error estimate at $t_{j+1}$ is given by

$$
\left\|E_{j+1}\right\| \leq C(\Delta t)^{2}, \quad j=1,2, \ldots, M-1 .
$$

Proof. Using the local error estimate up to the $(j+1)$ th time step given in the above lemma, we obtain the global error estimate at the $(j+1)$ th time step as

$$
\begin{aligned}
\left\|E_{j+1}\right\| & =\left\|\sum_{l=1}^{j+1} e_{l}\right\| \\
& \leq\left\|e_{1}\right\|+\left\|e_{2}\right\|+\cdots+\left\|e_{j+1}\right\| \\
& \leq C_{1}(j \Delta t)(\Delta t)^{2} \\
& \leq C_{1} T(\Delta t)^{2}, \text { since }(j+1) \Delta t \leq T \\
& =C(\Delta t)^{2}, C_{1} T=C,
\end{aligned}
$$

where $C$ is constant independent of $c_{\varepsilon}$ and $\Delta t$.

Next, we set a bound for the derivatives of solution of the problem in (15).

Lemma 8. For each, $j=0,1, \ldots, M-1$, the solution of the boundary value problems in (15) satisfies the following bound:

$$
\begin{array}{r}
\left|\frac{d^{k}}{\mathrm{~d} x^{k}} U_{j+1}(x)\right| \leq C\left(1+c_{\varepsilon}^{-k} \exp \left(-\frac{p^{*}(1-x)}{c_{\varepsilon}}\right)\right), \\
x \in \bar{\Omega}, 0 \leq k \leq 4 .
\end{array}
$$

Proof. See in [23].

4.2. Spatial Discretization. For the spatial discretization, we apply the nonstandard FDM. The construction of nonstandard FDM depends on the knowledge of the corresponding exact FDM, which is given in [24].

4.2.1. Exact Finite Difference Method. For the problem of the form in (15), in order to construct exact finite difference scheme, we follow the procedures developed in [24] for BVPs. Consider the constant coefficient subequations from (15) as

$$
\begin{array}{r}
-c_{\varepsilon}\left(U_{j+1}(x)\right)_{x x}+p^{*}\left(U_{j+1}(x)\right)_{x}+2 \theta U_{j+1}(x)=0, \\
-c_{\varepsilon}\left(U_{j+1}(x)\right)_{x x}+p^{*}\left(U_{j+1}(x)\right)_{x}=0,
\end{array}
$$

where $p(x) \geq p^{*}$ and $d(x) \geq 2 \theta$. Since equation (27) is constant coefficient second-order differential equations, it has two independent solutions, that is,

$$
U_{j+1}(x)=A_{1} \exp \left(\lambda_{1} x\right)+A_{2} \exp \left(\lambda_{2} x\right),
$$

where

$$
\lambda_{1}=-p^{*}+\sqrt{\left(p^{*}\right)^{2}+8 c_{\varepsilon} \theta} /-2 c_{\varepsilon} \text { and } \lambda_{2}=
$$
$-p^{*}-\sqrt{\left(p^{*}\right)^{2}+8 c_{\varepsilon} \theta} /-2 c_{\varepsilon}$.

We discretize the spatial domain $[0,1]$, using uniform mesh length $\Delta x=h$ such that $\Omega^{N}=\left\{x_{i}=x_{0}+i h, i=1,2, \ldots\right.$, $\left.N, x_{0}=0, x_{N}=1, h=1 / N\right\}$, where $N$ is the number of mesh intervals in spatial discretization. We denote the approximate solution of $U_{j+1}(x)$ at mesh point $x_{i}$ by $U_{i, j+1}$. The target is to calculate a difference equation, which has the same general solution as (27) at the mesh point $x_{i}$ given by

$$
U_{i, j+1}=A_{1} \exp \left(\lambda_{1} x_{i}\right)+A_{2} \exp \left(\lambda_{2} x_{i}\right)
$$

Using the theory of difference equations for secondorder linear difference equations in [24], we obtain

$$
\left|\begin{array}{ccc}
U_{i-1, j+1} & \exp \left(\lambda_{1} x_{i-1}\right) & \exp \left(\lambda_{2} x_{i-1}\right) \\
U_{i, j+1} & \exp \left(\lambda_{1} x_{i}\right) & \exp \left(\lambda_{2} x_{i}\right) \\
U_{i+1, j+1} & \exp \left(\lambda_{1} x_{i+1}\right) & \exp \left(\lambda_{2} x_{i+1}\right)
\end{array}\right|=0
$$

and then, by simplifying and substituting the values of $\lambda_{1}$ and $\lambda_{2}$, we obtain 


$$
\begin{aligned}
& \exp \left(\frac{p^{*} h}{2 c_{\varepsilon}}\right) U_{i-1, j+1}-2 \cosh \left(\frac{h \sqrt{\left(p^{*}\right)^{2}+8 c_{\varepsilon} \theta}}{2 c_{\varepsilon}}\right) U_{i, j+1} \\
& +\exp \left(-\frac{p^{*} h}{2 c_{\varepsilon}}\right) U_{i+1, j+1}=0
\end{aligned}
$$

which is an exact difference scheme for (44). For $\varepsilon \longrightarrow 0$, we use the approximation $h \sqrt{\left(p^{*}\right)^{2}+8 c_{\varepsilon} \theta} / 2 c_{\varepsilon} \approx p^{*} h / 2 c_{\varepsilon}$ in (32). Hence, multiplying both sides by $\exp \left(p^{*} h / 2 c_{\varepsilon}\right)$, simplifying we obtain

$$
U_{i-1, j+1}-2 U_{i, j+1}+U_{i+1, j+1}=\left(\exp \left(\frac{p^{*} h}{c_{\varepsilon}}\right)-1\right)\left(U_{i, j+1}-U_{i-1, j+1}\right) .
$$

Rearranging, we obtain

$$
-c_{\varepsilon} \frac{U_{i-1, j+1}-2 U_{i, j+1}+U_{i+1, j+1}}{h c_{\varepsilon} / p^{*}\left(\exp \left(p^{*} h / c_{\varepsilon}\right)-1\right)}+p^{*} \frac{U_{i, j+1}-U_{i-1, j+1}}{h}=0 .
$$

The denominator function for the second-order derivative approximation in (34) is $\gamma^{2}:=h c_{\varepsilon} / p^{*}$ $\left(\exp \left(h p^{*} / c_{\varepsilon}\right)-1\right)$. For the variable coefficient problems, we modify the denominator function as

$$
\gamma_{i}^{2}=\frac{h c_{\varepsilon}}{p\left(x_{i}\right)}\left(\exp \left(\frac{h p\left(x_{i}\right)}{c_{\varepsilon}}\right)-1\right),
$$

where $\gamma_{i}^{2}$ is a function of $c_{\varepsilon}, p\left(x_{i}\right)$, and $h$.

4.3. The Discrete Scheme. Using the denominator function in (35) into the discretization of (15), we obtain the nonstandard finite difference schemes as

$$
L_{c_{\varepsilon}}^{h, \Delta t} U_{i, j+1}=g_{i, j+1}, \quad i=1,2, \ldots, N-1,
$$

where $\quad L_{c_{\varepsilon}, \Delta t}^{h,} U_{i, j+1}=-\left(c_{\varepsilon} / 2\right)\left(\left(U_{i+1, j+1}-2 U_{i, j+1}+U_{i-1, j+1}\right) /\right.$ $\left.\gamma_{i}^{2}\right)+\left(p\left(x_{i}\right) / 2\right)\left(\left(U_{i, j+1}-U_{i-1, j+1}\right) / h\right)+d\left(x_{i}\right) U_{i, j+1}$ and

$$
\begin{aligned}
g_{i, j+1}= & \frac{c_{\varepsilon}}{2} \frac{U_{i+1, j}-2 U_{i, j}+U_{i-1, j}}{\gamma_{i}^{2}}-\frac{p\left(x_{i}\right)}{2} \frac{U_{i, j}-U_{i-1, j}}{h} \\
& -r\left(x_{i}\right) U_{i, j}+\frac{1}{2}\left[f\left(x_{i}, t_{j+1}\right)+f\left(x_{i}, t_{j}\right)\right] .
\end{aligned}
$$

4.4. Stability and Uniform Convergence Analysis. In this subsection, we want to show that the discrete scheme in (24) satisfies the discrete maximum principle, uniform stability estimates. and uniform convergence.

Lemma 9. Discrete maximum principle. Let $U_{i, j+1}$ be any mesh function satisfying $U_{0, J+1} \geq 0, U_{N, j+1} \geq 0$. Then, $L_{\mathcal{C}_{\varepsilon}}^{h, \Delta t} U_{i, j+1} \geq 0, i=1,2, \ldots, N-1$, which implies that $U_{i, j+1}^{\mathcal{c}_{\varepsilon}} \geq 0, i=0,1, \ldots, N$.

Proof. Suppose that there exists $k \in\{0,1, \ldots, N\}$ such that $U_{k, j+1}=\min _{0 \leq i \leq N} U_{i, j+1}<0$. From the assumption, it implies $k \neq\{0, N\}$. Also, we assume that $U_{k+1, j+1}-U_{k, j+1}>0$ and $U_{k, j+1}-U_{k-1, j+1}<0$. Now, we have

$$
\begin{aligned}
L_{c_{\varepsilon}}^{h, \Delta t} U_{k, j+1}= & -\frac{c_{\varepsilon}}{2} \frac{U_{k+1, j+1}-2 U_{k, j+1}+U_{k-1, j+1}}{\gamma_{k}^{2}} \\
& +\frac{p\left(x_{k}\right)}{2} \frac{U_{k, j+1}-U_{k-1, j+1}}{h}+d\left(x_{k}\right) U_{k, j+1},
\end{aligned}
$$

and using the assumptions made above, we obtain $L_{c_{c}}^{h, \Delta t} U_{k, j+1}<0$, for $k=1,2,3, \ldots, N-1$. Thus, the supposition $U_{i, j+1}<0$, for $i=0,1, \ldots, N$ is wrong. Hence, we obtain. $U_{i, j+1} \geq 0, i=0,1, \ldots, N$

Next, we want to prove that the scheme in (36) satisfies the uniform stability estimate.

Lemma 10. Uniform stability estimate. The solution $U_{i, j+1}$ of the discrete scheme in (24) satisfies the following bound:

$$
\left|U_{i, j+1}\right| \leq \frac{\left\|L_{\mathcal{c}_{\varepsilon}}^{h, \Delta t} U_{i, j+1}\right\|}{\zeta^{*}}+\max \left\{\left|\phi\left(0, t_{j+1}\right)\right|,\left|\psi\left(1, t_{j+1}\right)\right|\right\},
$$

where $\zeta^{*}$ is the lower bound of $d(x)$.

Proof. Let $\quad Q=\left\|L_{c_{\varepsilon}}^{h, \Delta t} U_{i, j+1}\right\| / \zeta^{*}+\max \left\{\left|\phi\left(0, t_{j+1}\right)\right|\right.$, $\left.\left|\psi\left(1, t_{j+1}\right)\right|\right\}$ and define the barrier function $\vartheta_{i, j+1}^{ \pm}$by $\vartheta_{i, j+1}^{ \pm}=Q \pm U_{i, j+1}$. At the boundary points, we obtain

$$
\begin{gathered}
\vartheta_{0, j+1}^{ \pm}=Q \pm U_{0, j+1}=\frac{\left\|L_{\mathcal{c}_{\varepsilon}}^{h, \Delta t} U_{i, j+1}\right\|}{\zeta^{*}}+\max \left\{\left|\phi\left(0, t_{j+1}\right)\right|,\left|\psi\left(1, t_{j+1}\right)\right|\right\} \pm \phi\left(0, t_{j+1}\right) \geq 0, \\
\mathcal{\vartheta}_{N, j+1}^{ \pm}=Q \pm U_{N, j+1}=\frac{\left\|L_{\mathcal{c}_{\varepsilon}}^{h, \Delta t} U_{i, j+1}\right\|}{\zeta^{*}}+\max \left\{\left|\phi\left(0, t_{j+1}\right)\right|,\left|\psi\left(1, t_{j+1}\right)\right|\right\} \pm \psi\left(1, t_{j+1}\right) \geq 0 .
\end{gathered}
$$


On the discretized spatial domain $x_{i}, 0<i<N$, we obtain

$$
\begin{aligned}
& L_{c_{\varepsilon}}^{h, \Delta t} \vartheta_{i, j+1}^{ \pm}=-\frac{c_{\varepsilon}}{2}\left(\frac{Q \pm U_{i+1, j+1}-2\left(Q \pm U_{i, j+1}\right)+Q \pm U_{i-1, j+1}}{\gamma_{i}^{2}}\right) \\
&+\frac{p\left(x_{i}\right)}{2}\left(\frac{Q \pm U_{i, j+1}-Q \pm U_{i-1, j+1}}{h}\right)+d\left(x_{i}\right)\left(Q \pm U_{i, j+1}\right) \\
&= d\left(x_{i}\right) Q \pm L_{c_{\varepsilon}}^{h, \Delta t} U_{i, j+1} \\
&= d\left(x_{i}\right)\left(\frac{\left\|L_{c_{\varepsilon}}^{h, \Delta t} U_{i, j+1}\right\|}{\zeta^{*}}+\max \left\{\left|\phi\left(0, t_{j+1}\right)\right|,\left|\psi\left(1, t_{j+1}\right)\right|\right\}\right) \pm g_{i, j+1} \\
& \geq 0, \operatorname{since} d\left(x_{i}\right) \geq \zeta^{*} .
\end{aligned}
$$

Using the maximum principle in Lemma 9, we obtain $\vartheta_{i, j+1}^{ \pm} \geq 0, \forall x_{i} \in \bar{\Omega}^{N}$. Hence, the required bound is obtained.

Lemma 11. Let $\gamma_{i}^{2}$ be the denominator function defined in (35), and then, the following bound holds

$$
c_{\varepsilon}\left|\frac{h^{2}}{\gamma_{i}^{2}}-1\right| \leq C_{3} h \text {. }
$$

Proof. Let us define $\rho=p\left(x_{i}\right) h / c_{\varepsilon}, \rho \in(0, \infty)$. Using the expression for $\gamma_{i}^{2}$, we obtain

$$
\begin{aligned}
c_{\varepsilon}\left|\frac{h^{2}}{\gamma_{i}^{2}}-1\right| & =p\left(x_{i}\right) h\left|\frac{1}{\exp (\rho)-1}-\frac{1}{\rho}\right| \\
& =h p\left(x_{i}\right)\left(\frac{\exp (\rho)-1-\rho}{\rho(\exp (\rho)-1)}\right)=: h p\left(x_{i}\right) R(\rho) .
\end{aligned}
$$

Next, let us set a bound for $R(\rho)=\exp (\rho)-1-$ $\rho / \rho(\exp (\rho)-1)$. Since

$$
\begin{aligned}
& \lim _{\rho \longrightarrow 0} R(\rho)=\frac{1}{2}, \\
& \lim _{\rho \longrightarrow \infty} R(\rho)=0 .
\end{aligned}
$$

We obtain the bounds as $R(\rho) \leq C_{3}, \forall \rho \in(0, \infty)$. Hence, from (43) and (44), we obtain

$$
c_{\varepsilon}\left|\frac{h^{2}}{\gamma_{i}^{2}}-1\right| \leq C_{3} h
$$

Let us define the forward and backward finite differences operators in spatial direction as

$$
\begin{aligned}
& D_{x}^{+} Z_{j+1}\left(x_{i}\right)=\frac{Z_{j+1}\left(x_{i+1}\right)-Z_{j+1}\left(x_{i}\right)}{h}, \\
& D_{x}^{-} Z_{j+1}\left(x_{i}\right)=\frac{Z_{j+1}\left(x_{i}\right)-Z_{j+1}\left(x_{i-1}\right)}{h},
\end{aligned}
$$

respectively, and the second-order finite difference operator as

$$
\begin{aligned}
D_{x}^{+} D_{x}^{-} Z_{j+1}\left(x_{i}\right) & =\frac{D^{+} Z_{j+1}\left(x_{i}\right)-D^{-} Z_{j+1}\left(x_{i}\right)}{h} \\
& =\frac{Z_{j+1}\left(x_{i+1}\right)-2 Z_{j+1}\left(x_{i}\right)+Z_{j+1}\left(x_{i}\right)}{h^{2}} .
\end{aligned}
$$

Theorem 1. The discrete solution $U_{i, j+1}$ of the problem in (9) satisfies the truncation error bound:

$$
\left|L_{c_{\varepsilon}}^{h, \Delta t}\left(U_{j+1}\left(x_{i}\right)-U_{i, j+1}\right)\right| \leq C h\left(1+\max _{x_{i} \in(0,1)} \frac{\exp \left(-\left(p^{*}\left(1-x_{i}\right) / c_{\varepsilon}\right)\right)}{c_{\varepsilon}^{3}}\right) .
$$

Proof. We consider the truncation error

$$
\begin{aligned}
\left|L_{c_{\varepsilon}}^{h, \Delta t}\left(U_{j+1}\left(x_{i}\right)-U_{i, j+1}\right)\right|= & \left|-c_{\varepsilon}\left(\frac{d^{2}}{\mathrm{~d} x^{2}}-\frac{D_{x}^{+} D_{x}^{-} h^{2}}{\gamma_{i}^{2}}\right) U_{j+1}\left(x_{i}\right)+p\left(x_{i}\right)\left(\frac{d}{\mathrm{~d} x}-D_{x}^{-}\right) U_{j+1}\left(x_{i}\right)\right| \\
\leq & C_{1} c_{\varepsilon}\left|\left(\frac{d^{2}}{\mathrm{~d} x^{2}}-D_{x}^{+} D_{x}^{-}\right) U_{j+1}\left(x_{i}\right)\right|+C_{1} c_{\varepsilon}\left|\left(\frac{h^{2}}{\gamma_{i}^{2}}-1\right) D_{x}^{+} D_{x}^{-} U_{j+1}\left(x_{i}\right)\right| \\
& +C_{1} h\left|\frac{d^{2}}{\mathrm{~d} x^{2}} U_{j+1}\left(x_{i}\right)\right| .
\end{aligned}
$$


Using the estimate in Lemma 11 for the bound of $c_{\varepsilon}\left|\left(h^{2} / \gamma_{i}^{2}\right)-1\right|$, the truncation error bound becomes

$$
\left|L_{c_{\varepsilon}}^{h, \Delta t}\left(U_{j+1}\left(x_{i}\right)-U_{i, j+1}\right)\right| \leq C_{3} c_{\varepsilon} h^{2}\left|\frac{d^{4}}{\mathrm{~d} x^{4}} U_{j+1}\left(x_{i}\right)\right|+C_{3} h\left|\frac{d^{2}}{\mathrm{~d} x^{2}} U_{j+1}\left(x_{i}\right)\right| \text {. }
$$

Using the bound of the derivatives of the solution in Lemma 8 in (50) gives

$$
\begin{aligned}
\left|L_{c_{\varepsilon}}^{h, \Delta t}\left(U_{j+1}\left(x_{i}\right)-U_{i, j+1}\right)\right| & \leq C_{4} h^{2}\left|c_{\varepsilon}+c_{\varepsilon}^{-3} \exp \left(-\frac{p^{*}\left(1-x_{i}\right)}{c_{\varepsilon}}\right)\right|+C_{4} h\left|1+c_{\varepsilon}^{-2} \exp \left(-\frac{p^{*}\left(1-x_{i}\right)}{c_{\varepsilon}}\right)\right| \\
& \leq C h\left(1+\max _{x_{i} \in(0,1)} \frac{\exp \left(-\left(p^{*}\left(1-x_{i}\right) / c_{\varepsilon}\right)\right)}{c_{\varepsilon}^{3}}\right), \text { since, } c_{\varepsilon}^{3} \leq c_{\varepsilon}^{2} .
\end{aligned}
$$

This completes the proof.

Lemma 12. For a fixed mesh number $N$ and for $\varepsilon \longrightarrow 0$, it holds

$$
\lim _{c_{\varepsilon} \longrightarrow 0} \max _{1 \leq i \leq N-1} \frac{\exp \left(-\left(p^{*}\left(1-x_{i}\right) / c_{\varepsilon}\right)\right)}{c_{\varepsilon}^{m}}=0, \quad m=1,2,3, \ldots,
$$

where $x_{i}=i h, h=1 / N, \forall i=1,2, \ldots, N-1$.

Proof. Refer in $[25,26]$.

Theorem 2. The discrete solution $U_{i, j+1}$ of the problem in (15) satisfies the error bound:

$$
\max _{x_{i} \in(0,1)}\left|U_{j+1}\left(x_{i}\right)-U_{i, j+1}\right| \leq C N^{-1},
$$

where $h=N^{-1}$.

Proof. By using the results of Lemma 12 in Theorem 1 and applying the bound in Lemma 10 gives the required bound.
Theorem 3. Under the hypothesis of boundedness of discrete solution, the solution of (7) and (8) satisfies the parameter uniform bound:

$$
\sup _{0<c_{\varepsilon} \ll 1} \max _{x_{i} \in(0,1)}\left|u\left(x_{i}, t_{j+1}\right)-U_{i, j+1}\right| \leq C\left(N^{-1}+(\Delta t)^{2}\right) .
$$

Proof. The parameter uniform convergence of the scheme follows from the bound in (15) and (31):

$$
E^{N, M}=\max _{\varepsilon, \delta, \eta}\left|E_{\varepsilon, \delta, \eta}^{N, M}\right| \text {. }
$$

\section{Numerical Results and Discussion}

To validate the established theoretical results, we develop an algorithm and perform experiments using the proposed numerical scheme on the problem of the form given in (2) and (4).

Example 1. Consider the problem

$$
\frac{\partial u}{\partial t}-\varepsilon^{2} \frac{\partial^{2} u}{\partial x^{2}}+\left(2-x^{2}\right) \frac{\partial u}{\partial x}+2 u(x-\delta, t)+(x-3) u(x, t)+u(x+\eta, t)=10 t^{2} \exp (-t) x(1-x)
$$

with the initial condition $u_{0}(x)=0,0 \leq x \leq 1$ and the interval conditions $\phi(x, t)=0,-\delta \leq x \leq 0, \psi(x, t)=0,1 \leq$ $x \leq 1+\eta$, and $T=3$.
Example 2. Consider the problem

$$
\frac{\partial u}{\partial t}-\varepsilon^{2} \frac{\partial^{2} u}{\partial x^{2}}+\left(2-x^{2}\right) \frac{\partial u}{\partial x}+(1+x) u(x-\delta, t)+\left(1+x^{2}+\cos (\pi x)\right) u(x, t)+3 u(x+\eta, t)=\sin (\pi x)
$$




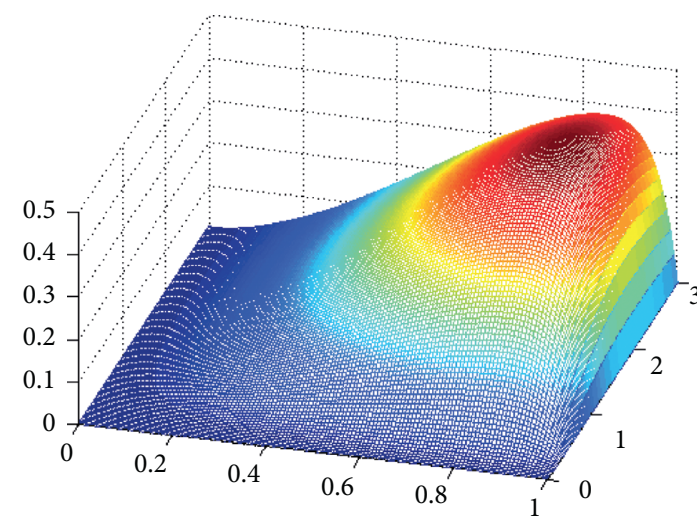

(a)

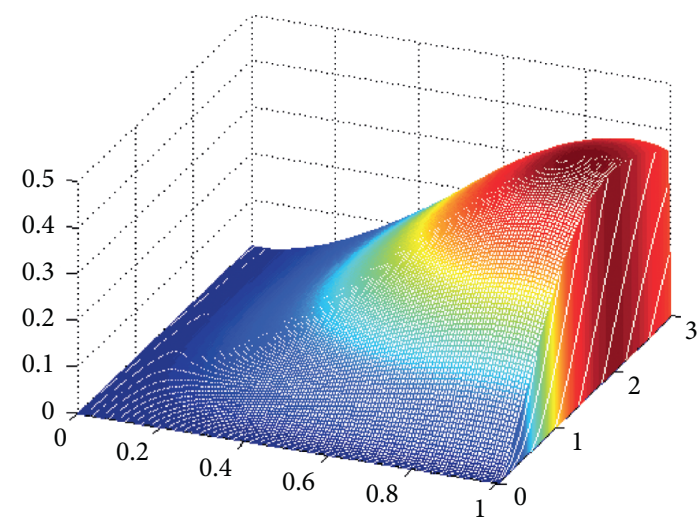

(b)

Figure 1: 3D view of numerical solution of example 1 on (a) $\varepsilon=2^{-2}$ on (b) $\varepsilon=2^{-20}$ for $\delta=0.6 \varepsilon, \eta=0.5 \varepsilon$.

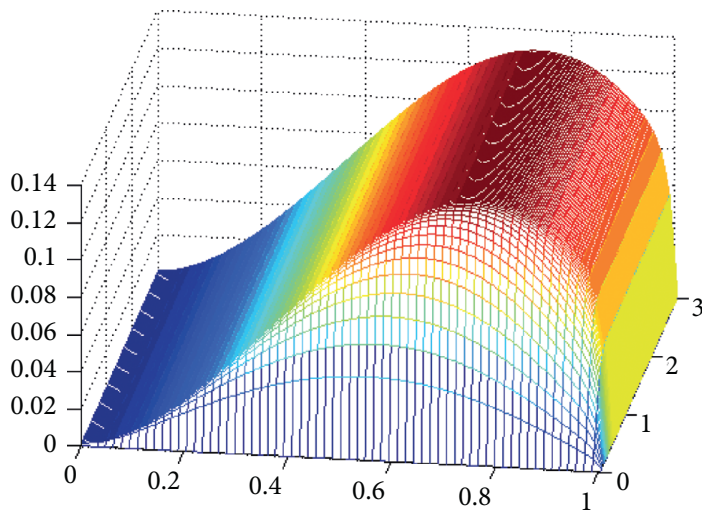

(a)

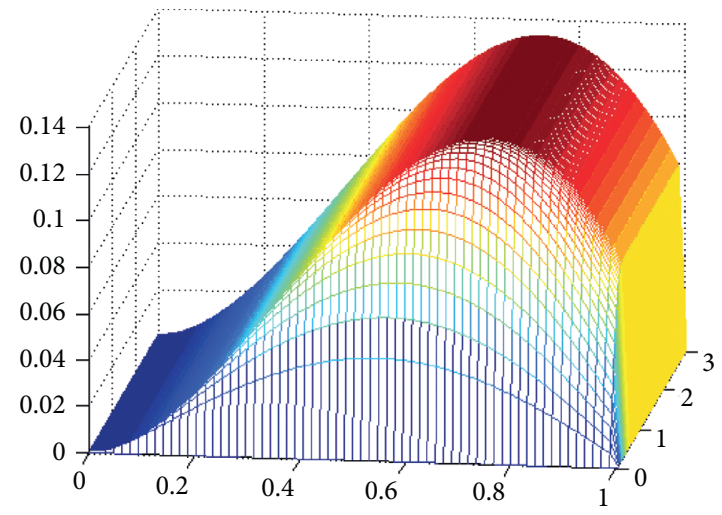

(b)

FIgURE 2: 3D view of numerical solution of example 2 exhibiting right boundary layer on (a) $\varepsilon=2^{-2}$ on $(\mathrm{b}) \varepsilon=2^{-20}$ for $\delta=0.6 \varepsilon, \eta=0.5 \varepsilon$.

with the initial condition $u_{0}(x)=0,0 \leq x \leq 1$ and the interval conditions $\phi(x, t)=0,-\delta \leq x \leq 0, \psi(x, t)=0,1 \leq$ $x \leq 1+\eta$, and $T=3$.

The exact solution of the considered examples is not available. So, the maximum absolute error, $\varepsilon$-uniform error and rate of convergence are calculated using the double mesh principle. Let $U_{i, j}^{N, M}$ be the computed solution of the problem using $N, M$ numbers of mesh points and $U_{i, j}^{2 N, 2 M}$ be the computed solution on the double number of mesh points $2 N, 2 M$ by including the midpoints $x_{i+1 / 2}$ and $t_{j+1 / 2}$ into the mesh points. The maximum absolute error is calculated as

$$
E_{\varepsilon, \delta, \eta}^{N, M}=\max _{i, j}\left|U_{i, j}^{N, M}-U_{i, j}^{2 N, 2 M}\right| .
$$

The uniform error estimate is calculated using

$$
E^{N, M}=\max _{\varepsilon, \delta, \eta}\left|E_{\varepsilon, \delta, \eta}^{N, M}\right| .
$$

The rate of convergence of the scheme is calculated using

$$
r_{\varepsilon, \delta, \eta}^{N, M}=\log _{2}\left(\frac{E_{\varepsilon, \delta, \eta}^{N, M}}{E_{\varepsilon, \delta, \eta}^{2 N, 2 M}}\right),
$$

and the uniform rate of convergence is calculated using

$$
r^{N, M}=\log _{2}\left(\frac{E^{N, M}}{E^{2 N, 2 M}}\right) .
$$

The solution of the problems in example 1 and example 2 exhibits a regular boundary layer on the right side of the spatial domain. In Figures 1 and 2, one can observe boundary layer formation as the perturbation parameter $\varepsilon$ goes small, particularly at $T=3$, one can observe in Figures 3(d) and 4(d). In Figures 3(a) $-3(\mathrm{~d})$ and 4(a) $-4(\mathrm{~d})$, the effect of the shift parameters and the perturbation parameter is shown on the profile of the solution by considering different values for the arameters.

The numerical result in Tables 1 and 2 show the maximum absolute error and rate of convergence of the scheme. The maximum absolute errors presented in Tables 1 and 3 


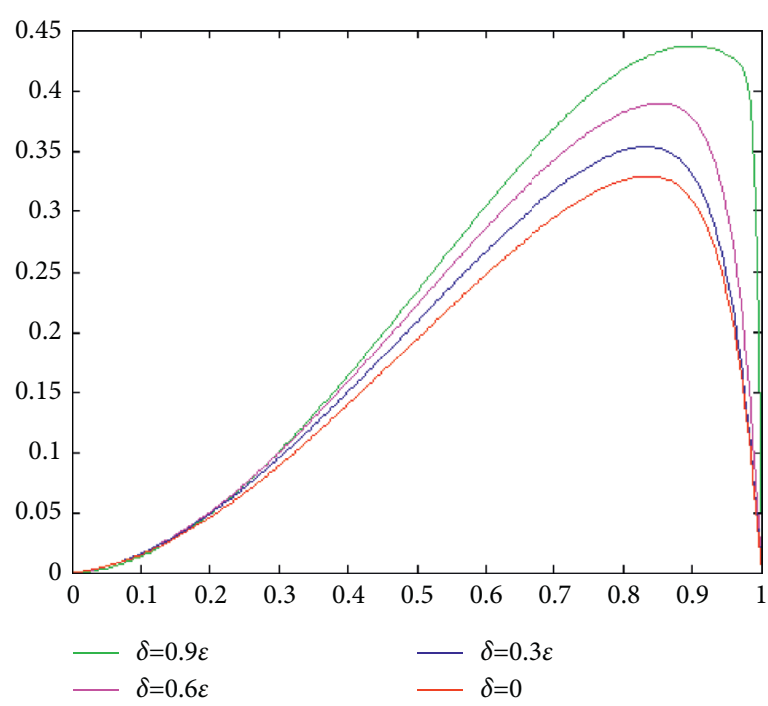

(a)

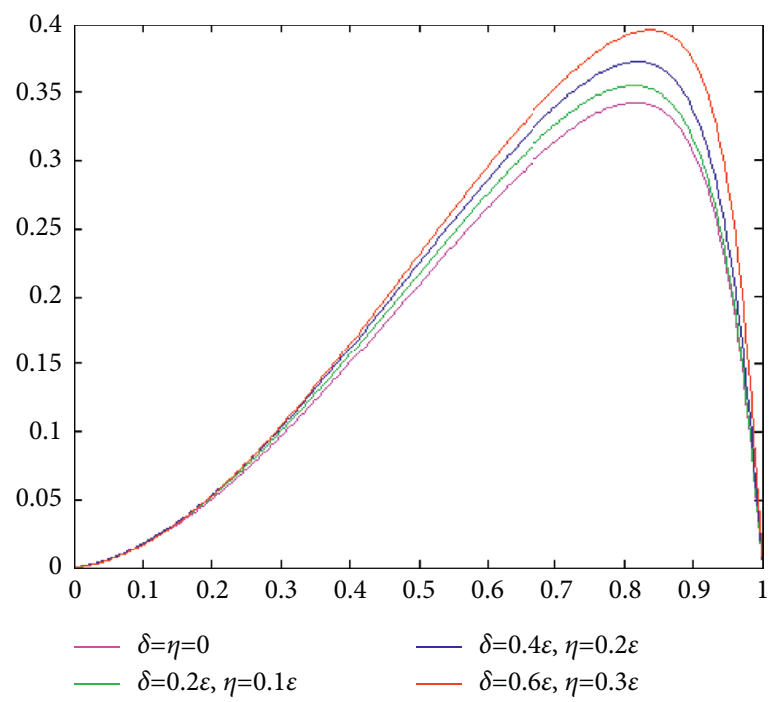

(c)

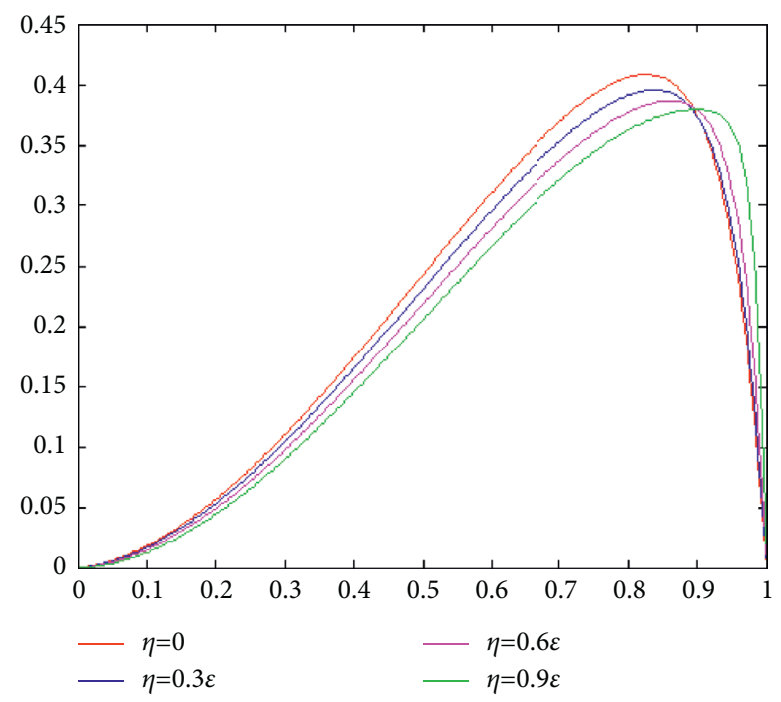

(b)

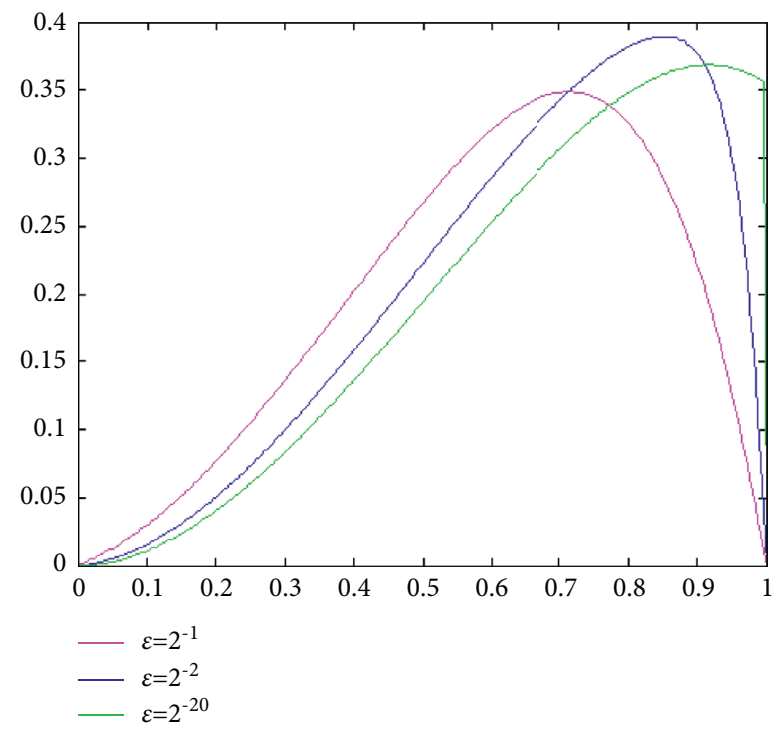

(d)

FIGURE 3: Example 1, on (a) effect of delay, (b) effect of advance, (c) effect of both advance and delay, and (d) effect of perturbation parameter on solution at $T=3$.

show the uniform convergence of the scheme. One can observe from these tables, as the value of the perturbation parameter goes small, the maximum absolute error becomes stable and constant (i.e., converges are independent of the influence of the perturbation parameters $\varepsilon$ ). Tables 3 and 4 show the maximum absolute error for different values of delay and advance parameters. As it is observed in these tables, the shift parameters do not influence the uniform convergence of the scheme. Table 5 shows the maximum absolute error and rate of convergence of the scheme for temporal discretization. As one observes in this table, the temporal discretization has the second order of convergence with correct agreement to the theoretical analysis. Tables 6 and 7 present the uniform error and uniform rate of convergence of the scheme and results from published papers in literature. The proposed scheme is more accurate than the upwind scheme in the Shishkin mesh, midpoint upwind scheme in the Shishkin mesh in [4], cubic B-spline scheme in the Shishkin mesh in [12], and the exponentially fitted scheme in [13].

Figure 5 shows the log-log plot of the maximum absolute error verses number of mesh points in space $N$ of the scheme for different values of the perturbation parameter. As one observes from the figures, for all values of the perturbation parameter, the line graphs are overlapped and parallel to the reference line $O\left(N^{-1}\right)$. This indicates that the convergence of the scheme is independent of the perturbation parameter $\varepsilon$. 


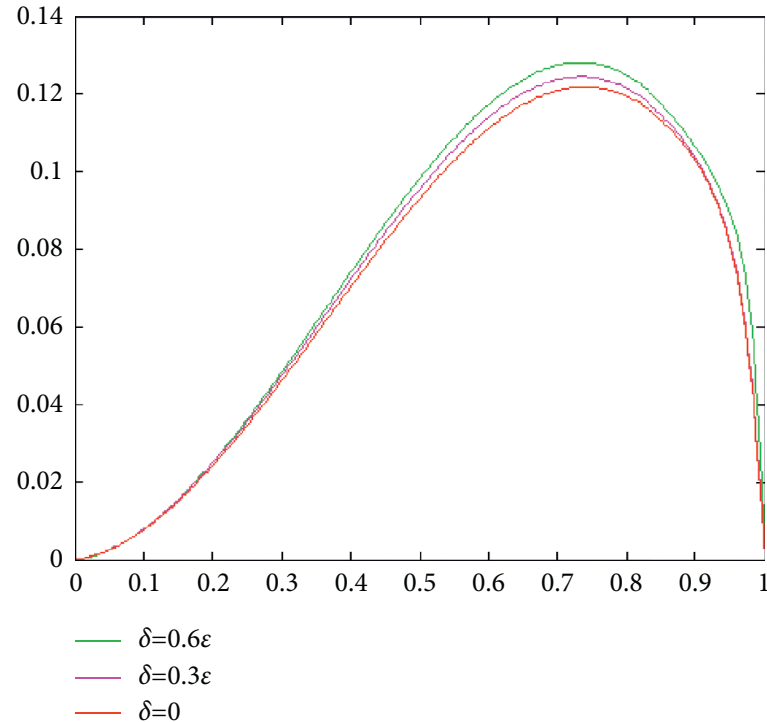

(a)

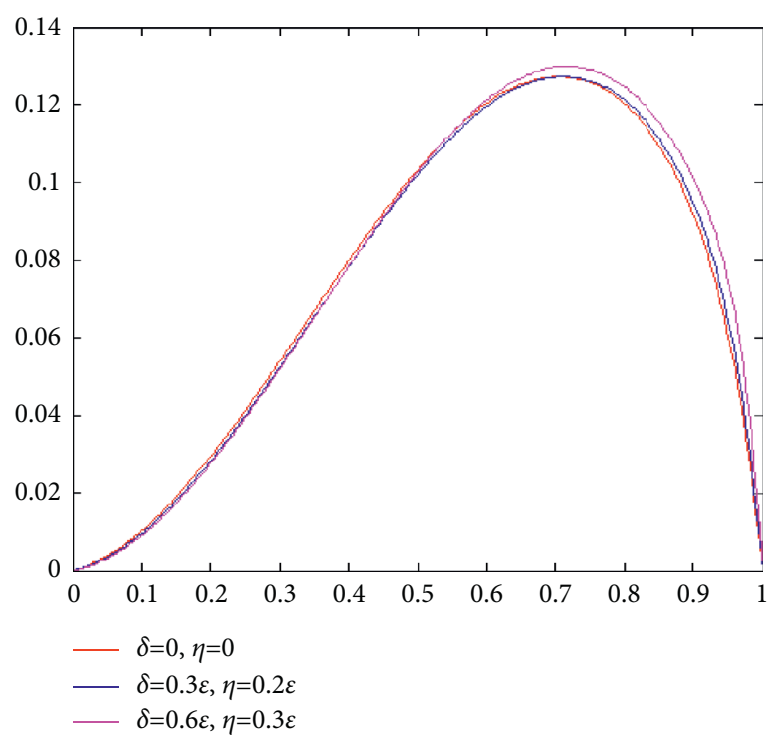

(c)

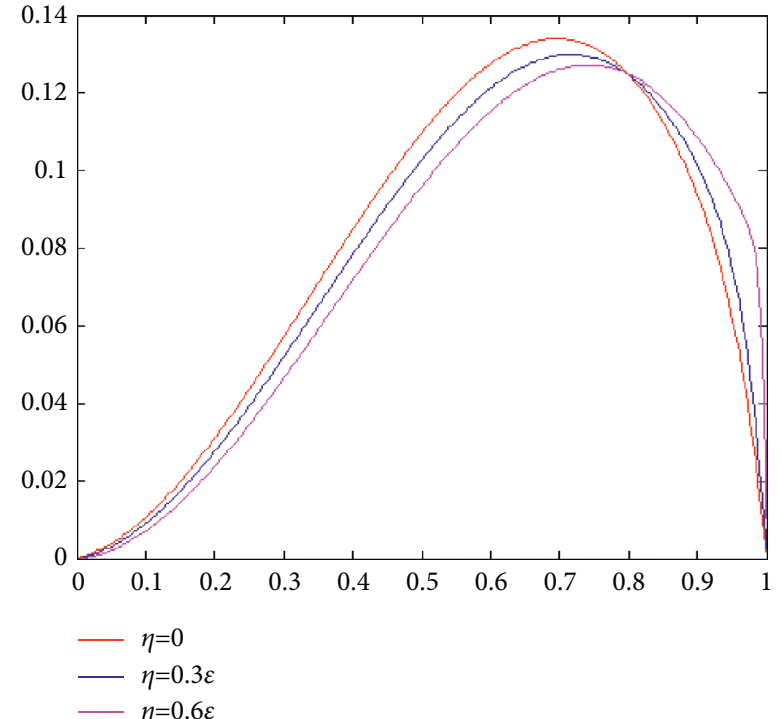

(b)

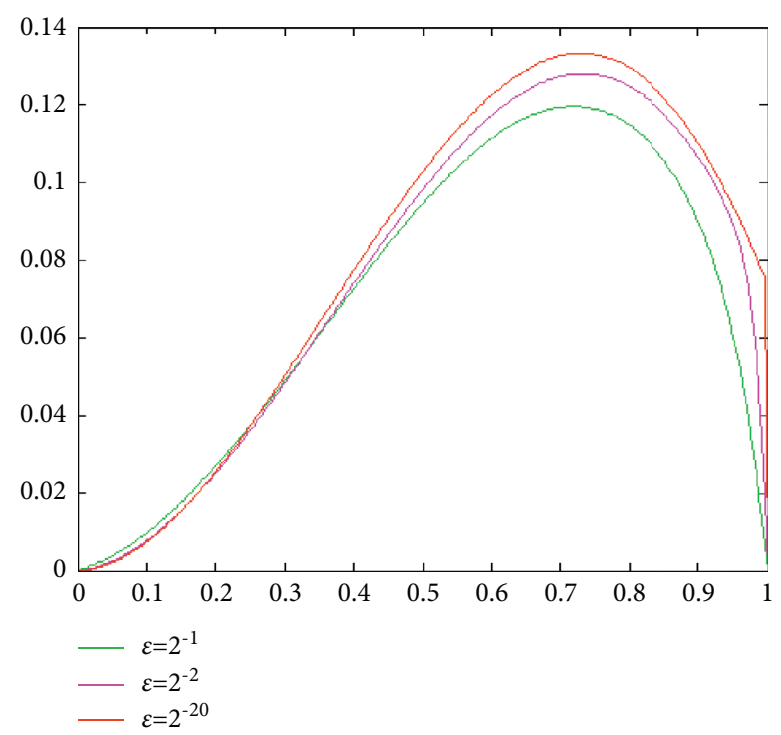

(d)

Figure 4: Example 2, on (a) effect of delay, (b) effect of advance, (c) effect of both advance and delay, and (d) effect of perturbation parameter on solution at $T=3$.

TABle 1: Example 1, maximum absolute error and rate of convergence for $\delta=0.6 \varepsilon$ and $\eta=0.5 \varepsilon$.

\begin{tabular}{|c|c|c|c|c|c|c|}
\hline$\varepsilon$ & $N=2^{5}$ & $2^{6}$ & $2^{7}$ & $2^{8}$ & $2^{9}$ & $2^{10}$ \\
\hline$\downarrow$ & $M=60$ & 120 & 240 & 480 & 960 & 1920 \\
\hline $2^{-6}$ & $\begin{array}{c}5.7729 e-03 \\
0.9785\end{array}$ & $\begin{array}{c}2.9297 e-03 \\
0.9893\end{array}$ & $\begin{array}{c}1.4758 e-03 \\
0.9946\end{array}$ & $\begin{array}{c}7.4067 e-04 \\
0.9973\end{array}$ & $\begin{array}{c}3.7103 e-04 \\
0.9996\end{array}$ & $\begin{array}{c}1.8557 e-04 \\
-\end{array}$ \\
\hline $2^{-8}$ & $\begin{array}{c}5.7496 e-03 \\
0.9785\end{array}$ & $\begin{array}{c}2.9179 e-03 \\
0.9892\end{array}$ & $\begin{array}{c}1.4699 e-03 \\
0.9946\end{array}$ & $\begin{array}{c}7.3769 e-04 \\
0.9973\end{array}$ & $\begin{array}{c}3.6953 e-04 \\
0.9986\end{array}$ & $\begin{array}{c}1.8494 e-04 \\
-\end{array}$ \\
\hline $2^{-10}$ & $\begin{array}{c}5.7439 e-03 \\
0.9785\end{array}$ & $\begin{array}{c}2.9150 e-03 \\
0.9893\end{array}$ & $\begin{array}{c}1.4684 e-03 \\
0.9946\end{array}$ & $\begin{array}{c}7.3694 e-04 \\
0.9973\end{array}$ & $\begin{array}{c}3.6916 e-04 \\
0.9987\end{array}$ & $\begin{array}{c}1.8475 e-04 \\
-\end{array}$ \\
\hline $2^{-12}$ & $\begin{array}{c}5.7424 e-03 \\
0.9786\end{array}$ & $\begin{array}{c}2.9142 e-03 \\
0.9892\end{array}$ & $\begin{array}{c}1.4680 e-03 \\
0.9946\end{array}$ & $\begin{array}{c}7.3676 e-04 \\
0.9973\end{array}$ & $\begin{array}{c}3.6906 e-04 \\
0.9987\end{array}$ & $\begin{array}{c}1.8470 e-04 \\
-\end{array}$ \\
\hline $2^{-14}$ & $\begin{array}{c}5.7421 e-03 \\
0.9785\end{array}$ & $\begin{array}{c}2.9141 e-03 \\
0.9893\end{array}$ & $\begin{array}{c}1.4679 e-03 \\
0.9946\end{array}$ & $\begin{array}{c}7.3671 e-04 \\
0.9973\end{array}$ & $\begin{array}{c}3.6904 e-04 \\
0.9987\end{array}$ & $\begin{array}{c}1.8469 e-04 \\
-\end{array}$ \\
\hline
\end{tabular}


TABLE 1: Continued.

\begin{tabular}{|c|c|c|c|c|c|c|}
\hline$\varepsilon$ & $N=2^{5}$ & $2^{6}$ & $2^{7}$ & $2^{8}$ & $2^{9}$ & $2^{10}$ \\
\hline$\downarrow$ & $M=60$ & 120 & 240 & 480 & 960 & 1920 \\
\hline $2^{-16}$ & $\begin{array}{c}5.7420 e-03 \\
0.9786\end{array}$ & $\begin{array}{c}2.9140 e-03 \\
0.9892\end{array}$ & $\begin{array}{c}1.4679 e-03 \\
0.9946\end{array}$ & $\begin{array}{c}7.3670 e-04 \\
0.9973\end{array}$ & $\begin{array}{c}3.6904 e-04 \\
0.9987\end{array}$ & $\begin{array}{c}1.8469 e-04 \\
-\end{array}$ \\
\hline $2^{-18}$ & $\begin{array}{c}5.7420 e-03 \\
0.9786\end{array}$ & $\begin{array}{c}2.9140 e-03 \\
0.9892\end{array}$ & $\begin{array}{c}1.4679 e-03 \\
0.9946\end{array}$ & $\begin{array}{c}7.3670 e-04 \\
0.9973\end{array}$ & $\begin{array}{c}3.6903 e-04 \\
0.9986\end{array}$ & $\begin{array}{c}1.8469 e-04 \\
-\end{array}$ \\
\hline $2^{-20}$ & $\begin{array}{c}5.7420 e-03 \\
0.9786\end{array}$ & $\begin{array}{c}2.9140 e-03 \\
0.9892\end{array}$ & $\begin{array}{c}1.4679 e-03 \\
0.9946\end{array}$ & $\begin{array}{c}7.3670 e-04 \\
0.9973\end{array}$ & $\begin{array}{c}3.6903 e-04 \\
0.9986\end{array}$ & $\begin{array}{c}1.8468 e-04 \\
-\end{array}$ \\
\hline $\begin{array}{l}E^{N, M} \\
r^{N, M}\end{array}$ & $\begin{array}{c}5.7729 e-03 \\
0.9785\end{array}$ & $\begin{array}{c}2.9297 e-03 \\
0.9892\end{array}$ & $\begin{array}{c}1.4758 e-03 \\
0.9946\end{array}$ & $\begin{array}{c}7.4067 e-04 \\
0.9973\end{array}$ & $\begin{array}{c}3.7103 e-04 \\
0.9987\end{array}$ & $\begin{array}{c}1.8557 e-04 \\
-\end{array}$ \\
\hline
\end{tabular}

TABLE 2: Example 2, maximum absolute error and rate of convergence for $\delta=0.6 \varepsilon$ and $\eta=0.5 \varepsilon$.

\begin{tabular}{|c|c|c|c|c|c|c|}
\hline$\varepsilon$ & $N=2^{4}$ & $2^{5}$ & $2^{6}$ & $2^{7}$ & $2^{8}$ & $2^{9}$ \\
\hline$\downarrow$ & $M=30$ & 60 & 120 & 240 & 480 & 960 \\
\hline $2^{-6}$ & $\begin{array}{c}3.9860 e-03 \\
0.9047\end{array}$ & $\begin{array}{c}2.1291 e-03 \\
0.9605\end{array}$ & $\begin{array}{c}1.0941 e-03 \\
0.9804\end{array}$ & $\begin{array}{c}5.5455 e-04 \\
0.9899\end{array}$ & $\begin{array}{c}2.7923 e-04 \\
0.9950\end{array}$ & $\begin{array}{c}1.4010 e-04 \\
-\end{array}$ \\
\hline $2^{-8}$ & $\begin{array}{c}3.9858 e-03 \\
0.9054\end{array}$ & $\begin{array}{c}2.1279 e-03 \\
0.9610\end{array}$ & $\begin{array}{c}1.0931 e-03 \\
0.9801\end{array}$ & $\begin{array}{c}5.5416 e-04 \\
0.9899\end{array}$ & $\begin{array}{c}2.7902 e-04 \\
0.9950\end{array}$ & $\begin{array}{c}1.3999 e-04 \\
-\end{array}$ \\
\hline $2^{-10}$ & $\begin{array}{c}3.9857 e-03 \\
0.9056\end{array}$ & $\begin{array}{c}2.1276 e-03 \\
0.9611\end{array}$ & $\begin{array}{c}1.0929 e-03 \\
0.9800\end{array}$ & $\begin{array}{c}5.5406 e-04 \\
0.9899\end{array}$ & $\begin{array}{c}2.7897 e-04 \\
0.9950\end{array}$ & $\begin{array}{c}1.3997 e-04 \\
-\end{array}$ \\
\hline $2^{-12}$ & $\begin{array}{c}3.9857 e-03 \\
0.9057\end{array}$ & $\begin{array}{c}2.1275 e-03 \\
0.9611\end{array}$ & $\begin{array}{c}1.0928 e-03 \\
0.9800\end{array}$ & $\begin{array}{c}5.5404 e-04 \\
0.9899\end{array}$ & $\begin{array}{c}2.7896 e-04 \\
0.9950\end{array}$ & $\begin{array}{c}1.3996 e-04 \\
-\end{array}$ \\
\hline $2^{-14}$ & $\begin{array}{c}3.9857 e-03 \\
0.9057\end{array}$ & $\begin{array}{c}2.1275 e-03 \\
0.9611\end{array}$ & $\begin{array}{c}1.0928 e-03 \\
0.9800\end{array}$ & $\begin{array}{c}5.5403 e-04 \\
0.9900\end{array}$ & $\begin{array}{c}2.7895 e-04 \\
0.9950\end{array}$ & $\begin{array}{c}1.3996 e-04 \\
-\end{array}$ \\
\hline $2^{-16}$ & $\begin{array}{c}3.9857 e-03 \\
0.9057\end{array}$ & $\begin{array}{c}2.1275 e-03 \\
0.9611\end{array}$ & $\begin{array}{c}1.0928 e-03 \\
0.9800\end{array}$ & $\begin{array}{c}5.5403 e-04 \\
0.9900\end{array}$ & $\begin{array}{c}2.7895 e-04 \\
0.9950\end{array}$ & $\begin{array}{c}1.3996 e-04 \\
-\end{array}$ \\
\hline $2^{-18}$ & $\begin{array}{c}3.9857 e-03 \\
0.9057\end{array}$ & $\begin{array}{c}2.1274 e-03 \\
0.9611\end{array}$ & $\begin{array}{c}1.0928 e-03 \\
0.9800\end{array}$ & $\begin{array}{c}5.5403 e-04 \\
0.9900\end{array}$ & $\begin{array}{c}2.7895 e-04 \\
0.9950\end{array}$ & $\begin{array}{c}1.3996 e-04 \\
-\end{array}$ \\
\hline $2^{-20}$ & $\begin{array}{c}3.9857 e-03 \\
0.9057\end{array}$ & $\begin{array}{c}2.1274 e-03 \\
0.9611\end{array}$ & $\begin{array}{c}1.0928 e-03 \\
0.9800\end{array}$ & $\begin{array}{c}5.5403 e-04 \\
0.9900\end{array}$ & $\begin{array}{c}2.7895 e-04 \\
0.9950\end{array}$ & $\begin{array}{c}1.3996 e-04 \\
-\end{array}$ \\
\hline $\begin{array}{l}E^{N, M} \\
r^{N, M}\end{array}$ & $\begin{array}{c}3.9860 e-03 \\
0.9047\end{array}$ & $\begin{array}{c}2.1291 e-03 \\
0.96050\end{array}$ & $\begin{array}{c}1.0941 e-03 \\
0.9800\end{array}$ & $\begin{array}{c}5.5455 e-04 \\
0.9899\end{array}$ & $\begin{array}{c}2.7923 e-04 \\
0.99500\end{array}$ & $\begin{array}{c}1.4010 e-04 \\
-\end{array}$ \\
\hline
\end{tabular}

TABle 3: Example 1, maximum absolute error for different values of $\delta$ and $\eta$ for $\varepsilon=2^{-10}$.

\begin{tabular}{|c|c|c|c|c|c|}
\hline & $\begin{array}{l}N=2^{5} \\
M=60\end{array}$ & $\begin{array}{c}2^{6} \\
120\end{array}$ & $\begin{array}{c}2^{7} \\
240\end{array}$ & $\begin{array}{c}2^{8} \\
480\end{array}$ & $\begin{array}{c}2^{9} \\
960\end{array}$ \\
\hline \multicolumn{6}{|c|}{$\delta \downarrow, \eta=0.5 \varepsilon$} \\
\hline 0 & $5.7406 e-03$ & $2.9133 e-03$ & $1.4676 e-03$ & $7.3652 e-04$ & $3.6895 e-04$ \\
\hline $0.1 \varepsilon$ & $5.7411 e-03$ & $2.9136 e-03$ & $1.4677 e-03$ & $7.3659 e-04$ & $3.6898 e-04$ \\
\hline $0.3 \varepsilon$ & $5.7422 e-03$ & $2.9141 e-03$ & $1.4680 e-03$ & $7.3673 e-04$ & $3.6905 e-04$ \\
\hline $0.5 \varepsilon$ & $5.7433 e-03$ & $2.9147 e-03$ & $1.4683 e-03$ & $7.3687 e-04$ & $3.6912 e-04$ \\
\hline $0.7 \varepsilon$ & $5.7444 e-03$ & $2.9153 e-03$ & $1.4685 e-03$ & $7.3701 e-04$ & $3.6919 e-04$ \\
\hline \multicolumn{6}{|c|}{$\eta \downarrow, \delta=0.6 \varepsilon$} \\
\hline 0 & $5.7452 e-03$ & $2.9157 e-03$ & $1.4688 e-03$ & $7.3712 e-04$ & $3.6925 e-04$ \\
\hline $0.1 \varepsilon$ & $5.7450 e-03$ & $2.9155 e-03$ & $1.4687 e-03$ & $7.3708 e-04$ & $3.6923 e-04$ \\
\hline $0.3 \varepsilon$ & $5.7444 e-03$ & $2.9153 e-03$ & $1.4685 e-03$ & $7.3701 e-04$ & $3.6919 e-04$ \\
\hline $0.5 \varepsilon$ & $5.7439 e-03$ & $2.9150 e-03$ & $1.4684 e-03$ & $7.3694 e-04$ & $3.6916 e-04$ \\
\hline $0.7 \varepsilon$ & $5.7433 e-03$ & $2.9147 e-03$ & $1.4683 e-03$ & $7.3687 e-04$ & $3.6912 e-04$ \\
\hline
\end{tabular}


TABLe 4: Example 2, maximum absolute error for different values of $\delta$ and $\eta$ for $\varepsilon=2^{-10}$.

\begin{tabular}{|c|c|c|c|c|c|}
\hline & $\begin{array}{l}N=2^{5} \\
M=60\end{array}$ & $\begin{array}{c}2^{6} \\
120 \\
\end{array}$ & $\begin{array}{c}2^{7} \\
240\end{array}$ & $\begin{array}{c}2^{8} \\
480\end{array}$ & $\begin{array}{c}2^{9} \\
960\end{array}$ \\
\hline \multicolumn{6}{|c|}{$\delta \downarrow, \eta=0.5 \varepsilon$} \\
\hline 0 & $2.1276 e-03$ & $1.0930 e-03$ & $5.5407 e-04$ & $2.7898 e-04$ & $1.3997 e-04$ \\
\hline $0.1 \varepsilon$ & $2.1276 e-03$ & $1.0929 e-03$ & $5.5407 e-04$ & $2.7897 e-04$ & $1.3997 e-04$ \\
\hline $0.3 \varepsilon$ & $2.1276 e-03$ & $1.0929 e-03$ & $5.5407 e-04$ & $2.7897 e-04$ & $1.3997 e-04$ \\
\hline $0.5 \varepsilon$ & $2.1276 e-03$ & $1.0929 e-03$ & $5.5406 e-04$ & $2.7897 e-04$ & $1.3997 e-04$ \\
\hline $0.7 \varepsilon$ & $2.1275 e-03$ & $1.0929 e-03$ & $5.5406 e-04$ & $2.7897 e-04$ & $1.3997 e-04$ \\
\hline \multicolumn{6}{|c|}{$\eta \downarrow, \delta=0.6 \varepsilon$} \\
\hline 0 & $2.1274 e-03$ & $1.0928 e-03$ & $5.5403 e-04$ & $2.7895 e-04$ & $1.3995 e-04$ \\
\hline $0.1 \varepsilon$ & $2.1275 e-03$ & $1.0928 e-03$ & $5.5403 e-04$ & $2.7895 e-04$ & $1.3995 e-04$ \\
\hline $0.3 \varepsilon$ & $2.1275 e-03$ & $1.0929 e-03$ & $5.5405 e-04$ & $2.7896 e-04$ & $1.3997 e-04$ \\
\hline $0.5 \varepsilon$ & $2.1276 e-03$ & $1.0929 e-03$ & $5.5406 e-04$ & $2.7897 e-04$ & $1.3998 e-04$ \\
\hline $0.6 \varepsilon$ & $2.1276 e-03$ & $1.0929 e-03$ & $5.5407 e-04$ & $2.7899 e-04$ & $1.3999 e-04$ \\
\hline
\end{tabular}

TABle 5: Maximum absolute error and rate of convergence for $\delta=0.6 \varepsilon$ and $\eta=0.5 \varepsilon$.

\begin{tabular}{|c|c|c|c|c|c|c|}
\hline \multirow[b]{2}{*}{$\varepsilon$} & \multicolumn{6}{|c|}{ Example 1} \\
\hline & $N=2^{5}$ & $2^{7}$ & $2^{9}$ & $2^{5}$ & $2^{7}$ & $2^{9}$ \\
\hline$\downarrow$ & $M=60$ & 120 & 240 & 60 & 120 & 240 \\
\hline $2^{-10}$ & $\begin{array}{c}5.7439 e-03 \\
1.9678\end{array}$ & $\begin{array}{c}1.4684 e-03 \\
1.9919\end{array}$ & $\begin{array}{c}3.6916 e-04 \\
-\end{array}$ & $\begin{array}{c}2.1276 e-03 \\
1.9411 \\
\end{array}$ & $\begin{array}{c}5.5406 e-04 \\
1.9849\end{array}$ & $\begin{array}{c}1.3997 e-04 \\
-\end{array}$ \\
\hline $2^{-12}$ & $\begin{array}{c}5.7424 e-03 \\
1.9678\end{array}$ & $\begin{array}{c}1.4680 e-03 \\
1.9919\end{array}$ & $\begin{array}{c}3.6906 e-04 \\
-\end{array}$ & $\begin{array}{c}2.1274 e-03 \\
1.9411 \\
\end{array}$ & $\begin{array}{c}5.5403 e-04 \\
1.9850\end{array}$ & $\begin{array}{c}1.3996 e-04 \\
-\end{array}$ \\
\hline $2^{-14}$ & $\begin{array}{c}5.7421 e-03 \\
1.9678\end{array}$ & $\begin{array}{c}1.4679 e-03 \\
1.9919\end{array}$ & $\begin{array}{c}3.6904 e-04 \\
-\end{array}$ & $\begin{array}{c}2.1274 e-03 \\
1.9411 \\
\end{array}$ & $\begin{array}{c}5.5403 e-04 \\
1.9850\end{array}$ & $\begin{array}{c}1.3996 e-04 \\
-\end{array}$ \\
\hline $2^{-16}$ & $\begin{array}{c}5.7420 e-03 \\
1.9678\end{array}$ & $\begin{array}{c}1.4679 e-03 \\
1.9919\end{array}$ & $\begin{array}{c}3.6904 e-04 \\
-\end{array}$ & $\begin{array}{c}.1274 e-03 \\
1.9411\end{array}$ & $\begin{array}{c}5.5403 e-04 \\
1.9850\end{array}$ & $\begin{array}{c}1.3996 e-04 \\
-\end{array}$ \\
\hline $2^{-18}$ & $\begin{array}{c}5.7420 e-03 \\
1.9678\end{array}$ & $\begin{array}{c}1.4679 e-03 \\
1.9919\end{array}$ & $\begin{array}{c}3.6903 e-04 \\
-\end{array}$ & $\begin{array}{c}2.1274 e-03 \\
1.9411 \\
\end{array}$ & $\begin{array}{c}5.5403 e-04 \\
1.9850\end{array}$ & $\begin{array}{c}1.3996 e-04 \\
-\end{array}$ \\
\hline $2^{-20}$ & $\begin{array}{c}5.7420 e-03 \\
1.9678\end{array}$ & $\begin{array}{c}1.4679 e-03 \\
1.9919\end{array}$ & $\begin{array}{c}3.6903 e-04 \\
-\end{array}$ & $\begin{array}{c}2.1274 e-03 \\
1.9411\end{array}$ & $\begin{array}{c}5.5403 e-04 \\
1.9850\end{array}$ & $\begin{array}{c}1.3996 e-04 \\
-\end{array}$ \\
\hline
\end{tabular}

TABLE 6: Example 1, comparison of uniform absolute error and uniform rate of convergence.

\begin{tabular}{|c|c|c|c|c|c|c|}
\hline Schemes & & $N=2^{6}$ & $2^{7}$ & $2^{8}$ & $2^{9}$ & $2^{10}$ \\
\hline$\downarrow$ & & $M=120$ & 240 & 480 & 960 & 1920 \\
\hline Proposed & $E^{N, M}$ & $2.9297 e-03$ & $1.4758 e-03$ & $7.4067 e-04$ & $3.7103 e-04$ & $1.8557 e-04$ \\
\hline Scheme & $r^{N, M}$ & 0.9892 & 0.9946 & 0.9973 & 0.9987 & - \\
\hline Upwind in [4] & $\begin{array}{l}E^{N, M} \\
r^{M, N}\end{array}$ & $\begin{array}{c}9.2021 e-03 \\
0.8840\end{array}$ & $\begin{array}{c}4.9863 e-03 \\
0.8912\end{array}$ & $\begin{array}{c}2.6885 e-03 \\
0.9163\end{array}$ & $\begin{array}{c}1.4245 e-03 \\
0.9178\end{array}$ & $\begin{array}{c}7.5403 e-04 \\
-\end{array}$ \\
\hline Midpoint up in [4] & $\begin{array}{l}E^{N, M} \\
r^{N, M}\end{array}$ & $\begin{array}{c}6.5942 e-03 \\
0.7877\end{array}$ & $\begin{array}{c}3.8199 e-03 \\
0.8508\end{array}$ & $\begin{array}{c}2.1180 e-03 \\
0.8938\end{array}$ & $\begin{array}{c}1.1399 e-03 \\
0.9107\end{array}$ & $\begin{array}{c}6.0635 e-04 \\
-\end{array}$ \\
\hline $\begin{array}{l}\text { B-spline } \\
\text { Colloc. in }[12]\end{array}$ & $\begin{array}{l}E^{N, M} \\
r^{N, M}\end{array}$ & $\begin{array}{c}4.4966 e-03 \\
0.8791\end{array}$ & $\begin{array}{c}2.4450 e-03 \\
0.9418\end{array}$ & $\begin{array}{c}1.2728 e-03 \\
0.9715\end{array}$ & $\begin{array}{c}6.4609 e-04 \\
0.9859\end{array}$ & $\begin{array}{c}3.2774 e-04 \\
-\end{array}$ \\
\hline Expo. & $E^{N, M}$ & $3.3107 e-03$ & $1.7254 e-03$ & $8.8049 e-04$ & $4.4473 e-04$ & $2.2349 e-04$ \\
\hline Fitted in [13] & $r^{N, M}$ & 0.9402 & 0.9705 & 0.9854 & 0.9927 & - \\
\hline
\end{tabular}

TABLE 7: Example 2, comparison of uniform absolute error and uniform rate of convergence.

\begin{tabular}{|c|c|c|c|c|c|c|}
\hline Schemes & & $N=2^{5}$ & $2^{6}$ & $2^{7}$ & $2^{8}$ & $2^{9}$ \\
\hline$\downarrow$ & & $M=60$ & 120 & 240 & 480 & 960 \\
\hline Proposed & $E^{N, M}$ & $2.1291 e-03$ & $1.0941 e-03$ & $5.5455 e-04$ & $2.7923 e-04$ & $1.4010 e-04$ \\
\hline Scheme & $r^{N, M}$ & 0.96050 & 0.9800 & 0.9899 & 0.99500 & - \\
\hline B-spline & $E^{N, M}$ & $7.6388 e-03$ & $3.8384 e-03$ & $1.9277 e-03$ & $9.6627 e-04$ & $4.8379 e-04$ \\
\hline Colloc. in [12] & $r^{N, M}$ & 0.9925 & 0.9925 & 0.9964 & 0.9981 & - \\
\hline
\end{tabular}




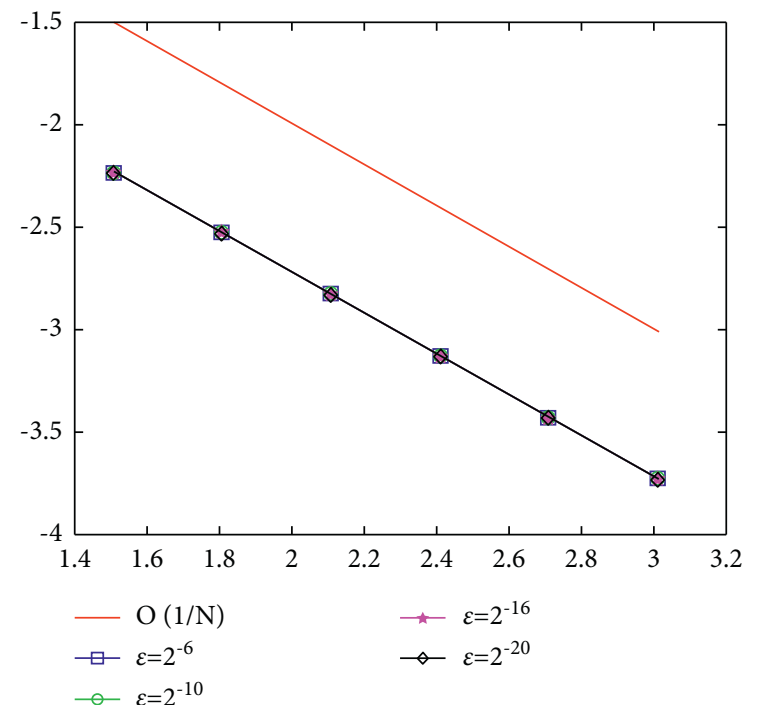

(a)

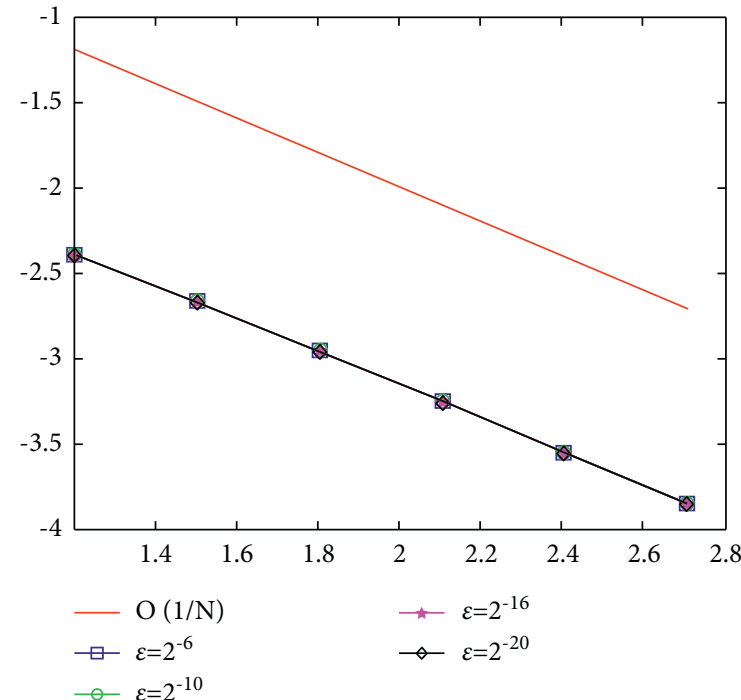

(b)

Figure 5: Log-log plot of maximum absolute error verses $N$ on (a) example 1 on (b) example 2 for different values of $\varepsilon$.

\section{Conclusion}

In this article, a parameter uniform numerical method is developed for solving a singularly perturbed parabolic differential equation involving both delay and advance on the spatial variable of reaction terms. The considered problems' solution exhibits a boundary layer on the right side of the spatial domain. The developed scheme constitutes the Crank-Nicolson method in temporal discretization and nonstandard FDM in the spatial discretization. The uniform stability and parameter uniform convergence of the scheme are investigated and proved. The applicability of the scheme is validated by taking numerical test examples. The effect of the perturbation parameter, effect of the delay, and advance parameters on the solution are depicted using figures. The proposed scheme converges uniformly (i.e., converges independent of the perturbation parameter) with the order of convergence one in space and two in time directions. The performance of the scheme is investigated by comparing the results with some schemes in published papers. We found that the proposed scheme is more accurate, stable, and parameter uniformly convergent.

\section{Data Availability}

No external data are used in this article.

\section{Conflicts of Interest}

The authors declare there are no potential conflicts of interest.

\section{References}

[1] Y. Kuang, Delay Differential Equations, University of California Press, Berkeley. CA, USA, 2012.
[2] M. K. Kadalbajoo and K. C. Patidar, "A survey of numerical techniques for solving singularly perturbed ordinary differential equations," Applied Mathematics and Computation, vol. 130, no. 2-3, pp. 457-510, 2002.

[3] M. K. Kadalbajoo and V. Gupta, "A brief survey on numerical methods for solving singularly perturbed problems," Applied Mathematics and Computation, vol. 217, no. 8, pp. 3641-3716, 2010.

[4] V. P. Ramesh and M. K. Kadalbajoo, "Upwind and midpoint upwind difference methods for time-dependent differential difference equations with layer behavior," Applied Mathematics and Computation, vol. 202, no. 2, pp. 453-471, 2008.

[5] R. Stein, "A theoretical analysis of neuronal variability," Biophysical Journal, vol. 5, no. 2, pp. 173-194, 1965.

[6] R. Stein, "Some models of neuronal variability," Biophysical Journal, vol. 7, no. 1, pp. 37-68, 1967.

[7] M. Musila and P. Lánskỳ, "Generalized Stein's model for anatomically complex neurons," Biosystems, vol. 25, no. 3, pp. 179-191, 1991.

[8] K. Bansal and K. K. Sharma, "Numerical technique for the class of time dependent singularly perturbed parabolic problems with state dependent retarded argument arising from generalised stein's model of neuronal variability," Journal of Dynamics and Differential Equations, pp. 1-28, 2016.

[9] K. Bansal and K. K. Sharma, "Parameter uniform numerical scheme for time dependent singularly perturbed convectiondiffusion-reaction problems with general shift arguments," Numerical Algorithms, vol. 75, no. 1, pp. 113-145, 2017.

[10] K. Bansal, P. Rai, and K. K. Sharma, "Numerical treatment for the class of time dependent singularly perturbed parabolic problems with general shift arguments," Journal of Dynamics and Differential Equations, vol. 25, no. 2, pp. 327-346, 2017.

[11] K. Bansal and K. K. Sharma, "Parameter-robust numerical scheme for time-dependent singularly perturbed reaction diffusion problem with large delay," Numerical Functional Analysis and Optimization, vol. 39, no. 2, pp. 127-154, 2018.

[12] D. Kumar and M. K. Kadalbajoo, "A parameter-uniform numerical method for time-dependent singularly perturbed 
differential-difference equations," Applied Mathematical Modelling, vol. 35, no. 6, pp. 2805-2819, 2011.

[13] N. Rao and P. Chakravarthy, "Fitted numerical methods for singularly perturbed $1 \mathrm{D}$ parabolic partial differential equations with small shifts arising in the modelling of neuronal variability," Differential Equations and Dynamical Systems, pp. 1-18, 2017.

[14] M. M. Woldaregay and G. F. Duressa, "Uniformly convergent numerical method for singularly perturbed delay parabolic differential equations arising in computational neuroscience," Kragujevac Journal of Mathematics, vol. 46, no. 1, pp. 65-84, 2022.

[15] MM. Woldaregay and GF. Duressa, "Fitted numerical scheme for solving singularly perturbed parabolic delay partial differential equations," Tamkang J. Math.vol. 53. , 2022 In press.

[16] MM. Woldaregay and GF. Duressa, "Uniformly convergent numerical scheme for singularly perturbed parabolic delay differential equations," ITM Web of Conferences, vol. 34, 2020.

[17] K. Kumar, PP. Chakravarthy, H. Ramos, and J. Vigo-Aguiar, "A stable finite difference scheme and error estimates for parabolic singularly perturbed PDEs with shift parameters," Journal of Computational and Applied Mathematics, pp. 1130-1150, 2020.

[18] M. Shivhare, PC. Podila, H. Ramos, and J. Vigo-Aguiar, "Quadratic B-spline collocation method for time dependent singularly perturbed differential-difference equation arising in the modeling of neuronal activity," Numerical Methods for Partial Differential Equations, 2021.

[19] IT. Daba and GF. Duressa, "Extended cubic B-spline collocation method for singularly perturbed parabolic differentialdifference equation arising in computational neuroscience," International Journal for Numerical Methods in Biomedical, vol. 37, no. 2, p. e3418, 2021.

[20] V. Gupta, M. Kumar, and S. Kumar, "Higher order numerical approximation for time dependent singularly perturbed differential-difference convection-diffusion equations," Numerical Methods for Partial Differential Equations, vol. 34, pp. 357-380, 2018.

[21] H. Tian, "The exponential asymptotic stability of singularly perturbed delay differential equations with a bounded lag," Journal of Mathematical Analysis and Applications, vol. 270, no. 1, pp. 143-149, 2002.

[22] H. G. Roos, M. Stynes, and L. Tobiska, Robust Numerical Methods for Singularly Perturbed Differential Equations, Springer Science \& Business Media, New York, NY, USA, 2008.

[23] C. Clavero, J. L. Gracia, and J. C. Jorge, "High order numerical methods for one dimensional parabolic singularly perturbed problems with regular layers," Numerical Methods for Partial Differential Equations, vol. 21, no. 1, pp. 149-169, 2005.

[24] E. R. Mickens, Advances in the Applications of Non-standard Finite Difference Schemes, World Scientific, Singapore, 2005.

[25] DA. Turuna, MM. Woldaregay, and GF. Duressa, "Uniformly convergent numerical method for singularly perturbed convection-diffusion problems," Kyungpook Mathematical Journal, vol. 60, no. 3, pp. 631-647, 2020.

[26] MM. Woldaregay, WT. Aniley, and GF. Duressa, "Novel numerical scheme for singularly perturbed time delay convection-diffusion equation," Advances in Theoretical and Mathematical Physics, vol. 2021, Article ID 6641236, 13 pages, 2021. 